\title{
A model for warm clouds with implicit droplet activation, avoiding saturation adjustment
}

https://doi.org/10.1515/mcwf-2018-0003

Received July 20, 2018; accepted December 9, 2018

Abstract: The representation of cloud processes in weather and climate models is crucial for their feedback on atmospheric flows. Since there is no general macroscopic theory of clouds, the parameterization of clouds in corresponding simulation software depends crucially on the underlying modeling assumptions. In this study we present a new model of intermediate complexity (a one-and-a-half moment scheme) for warm clouds, which is derived from physical principles. Our model consists of a system of differential-algebraic equations which allows for supersaturation and comprises intrinsic automated droplet activation due to a coupling of the droplet mass- and number concentrations tailored to this problem. For the numerical solution of this system we recommend a semi-implicit integration scheme, with efficient solvers for the implicit parts. The new model shows encouraging numerical results when compared with alternative cloud parameterizations, and it is well suited to investigate model uncertainties and to quantify predictability of weather events in moist atmospheric regimes.

Keywords: Cloud model, numerical methods

\section{Introduction}

Clouds are important components in the Earth-Atmosphere system. They influence the hydrological cycle via precipitation formation, the organization of weather phenomena (convection etc.), and the energy budget by their interaction with radiation. It is well known that clouds constitute a major source for forecast errors for weather prediction, or more precisely, they influence the predictability of moist atmospheric flows in a crucial way. This is mostly due to the fact that diabatic processes (e.g. latent heating or mixing) serve as large energy sources and sinks [e.g., 13, 14].

The representation of clouds in models for weather forecast and climate prediction is an important and challenging task. Cloud processes take place on a variety of scales and interact with other processes (e.g. atmospheric flows) in a truly multiscale fashion. Consisting of a myriad of small water particles which evolve in space and time, a rigorous simulation based on fundamental physical principles is way beyond current computing capacities. Standard implementations therefore resort to certain parameterizations of the cloud system; depending on the level of sophistication there exist (i) single moment schemes, which only keep track of the spatial water mass concentration for certain types of particles [e.g., 5, 15, 21], (ii) double moment schemes, which also monitor the number concentrations of these particles [e.g., 28, 37], and (iii) statistical models, which assume a statistical distribution of the particles over an admissible range of mass values, and then evolve the distribution function in time (and space), which leads to Boltzmann-type evolution equations [see, e.g., 2, 16, 17].

\footnotetext{
*Corresponding Author: Nikolas Porz: Institute for Atmospheric Physics, Johannes Gutenberg University, Mainz, Germany Martin Hanke: Institute of Mathematics, Johannes Gutenberg University, Mainz, Germany Manuel Baumgartner: Data Center, Johannes Gutenberg University, Mainz, Germany Peter Spichtinger: Institute for Atmospheric Physics, Johannes Gutenberg University, Mainz, Germany
} 
While the latter ansatz seems to be very attractive, there are several problems associated with it. First, at least to our knowledge, no consistent treatment of all cloud processes has yet been achieved with such a setting, although several attemps have been made in the literature: usually, these formulations concentrate on the collision terms but omit other important processes like, for example, particle formation [e.g., 2]. Second, it is often assumed that the type of the distribution does not change in time, but this assumption is violated for almost all important cloud processes [e.g., 6]. Third, even for an incomplete version of the corresponding evolution equations, their numerical treatment is quite difficult and expensive. Finally, measurements of the mass distribution of cloud particles are very difficult to realize, so that real data are lacking for model calibration.

On the other hand, real measurements are available for number and mass concentrations of the water droplets, i.e., for the corresponding variables of a two-moment scheme [e.g., 42]. In the statistical ansatz those correspond to certain moments of the distributions. We found that when focussing only on these number and mass concentrations, then a methodology which is well-known from chemical reaction networks and population dynamics leads essentially to the same dynamical system as when starting from a statistical description of the ensemble.

In the formulation of models based on averaged quantities like number and mass concentrations, the following problems need to be addressed:

1. Collision terms:

The formulation of collision terms is not straightforward, since the details and the evolution of the underlying size distribution must be mimicked in an adequate way. The standard separation of non-falling cloud droplets and falling rain drops due to [15] leads to artificial processes called autoconversion and accretion, which must be parameterized in a meaningful way.

2. Particle generation:

The formation of cloud droplets is based on the activation of cloud condensation nuclei (CCN) in the atmosphere [19]. For a proper treatment of this process, aerosols and their chemical and physical properties must be taken into account, which is difficult to model appropriately; it would require the extension of the model to also include aerosol physics.

3. Growth/evaporation of liquid droplets:

The representation of condensation processes can change the distribution of latent heating and thus influence the evolution of convective systems [e.g., 24]. Small cloud droplets grow exclusively by diffusion in the supersaturated regime, which is very fast for relevant temperatures; this leads to stiff differential equations and numerical instabilities. Many models therefore consider a technique known as saturation adjustment [e.g., 18], assuming water clouds at water saturation. However, this approach has a large effect on the vertical evolution of convection, since the latent heating is overestimated leading to significant errors in cloud buoyancy [cf. 7, 9, 10].

In addition to a consistent formulation of the processes and the model, we also need to discuss appropriate numerical schemes for solving the equations in an adequate way. Finally, we have to make sure that meaningful solutions exist.

Our motivation for the development of yet another cloud model based on bulk variables is as follows. To improve predictability of moist atmospheric flows, the adequate representation of clouds and their processes is an important issue. These investigations are pushed forward from the point of view of atmospheric dynamics and weather forecasts in connection with operational weather forecast models, as, e.g., COSMO or, more recent ICON, driven by the German Weather Service, or the IFS model used at the European Centre for Medium-Range Weather Forecasts. These operational models use very simple schemes for representing clouds. Usually, single moment schemes are used, which predict mass concentrations of cloud and rain water, only. It is well known that especially collision processes cannot be represented in a sufficient way for single moment schemes, which impedes an accurate prediction of the formation of rain. In addition, CCN activation cannot be treated well in such simple models; mostly it is assumed that clouds exist at thermodynamic equilibrium and the number of cloud droplets is prescribed. This gives rise to additional uncertainties and forecast errors. 
On the other hand, there are complex cloud models available for research purposes with sophisticated schemes for the treatment of collision processes and particle generation [see, e.g., 29, 30, 37]. However, it turns out that the investigation of such complex models and their impact on dynamics is very complicated; due to the complex and sometimes discrete formulation of the processes the estimation of the impact of these processes on dynamics is almost impossible.

As another important issue, the coefficients of many process rates in the governing equations are not well determined from first principles. Often, these rates are only known to belong to a certain range, but their exact values must be estimated by comparison with measurements or reference models. For the quantification of predictability of moist flows including clouds, we have to use inverse methods to investigate the uncertainties in these parameters first. For this reason it is desirable to have a model of only intermediate complexity.

In this study we develop such a model, well suited for a mathematical analysis of the associated processes and for the estimation of its parameters. To be precise, we develop a one-and-a-half moment scheme, i.e. a set of differential equations for mass concentration of cloud droplets and mass and number concentrations for rain drops. We solve the activation problem by introducing a functional relation between cloud droplet number and mass concentrations. Finally, cloud droplet growth and evaporation are treated using the diffusional growth equation, i.e., no saturation adjustment is used. For the proper treatment of these equations, we present a consistent sophisticated numerical scheme.

The paper is structured as follows: In the next section we describe the model including the relevant processes, the process rates, and their representation in mathematical terms. Subsequently, in Section 3, we develop our numerical scheme, and we present some numerical results in Section 4. We end the study with a short summary and some conclusions in Section 5.

\section{Model description}

Our model can be used as a box model for a single volume parcel or as a model of a vertical column of air, which is transported in a Lagrangian way (i.e., along a given trajectory). For the latter case we denote the vertical spatial coordinate by $z$. At the moment, the model is not coupled to any equations for atmospheric flows (i.e., Navier-Stokes equations, or relevant approximations), although this may be carried out in future work. A simpler version of our model was already implemented within a flow solver [23].

We restrict our model to so-called warm clouds or liquid clouds, which commonly occur in the temperature regime $250 \mathrm{~K}<T<310 \mathrm{~K}$, i.e., ice processes have not been included into the model. An extension into this direction is planned, but is beyond the scope of this work.

\subsection{Basic assumptions}

In warm clouds one can distinguish two water phases, namely water vapour and liquid droplets of various sizes. The droplets can interact with each other and also with water vapour, depending on the thermodynamic conditions (i.e., temperature, pressure, and water vapour concentration). Measurements indicate two well separated modes in the size/mass distribution of liquid water particles [41]. Therefore, we distinguish two species of water particles, namely cloud droplets (index $c$ ) and rain drops (index $r$ ), and as we have already said, we keep track of the bulk variables mass concentration $q_{x}$ and number concentration $n_{x}$ for each water particle species $x$, rather than their statistical distributions. To be precise, we only evolve number and mass concentrations for rain drops independently, whereas we couple the number concentration $n_{c}$ of cloud droplets to their mass concentration $q_{c}$ via a sophisticated functional relation, cf. (22).

As it is standard in cloud physics, number and mass concentrations are given in units per mass dry air, i.e., $\left[n_{x}\right]=\mathrm{kg}^{-1}$ and $\left[q_{x}\right]=\mathrm{kg} \mathrm{kg}^{-1}$. For simplification, we assume that cloud and rain drops are spherical, so that the mass $m$ of a water droplet (i.e., $m_{c}$ or $m_{r}$ ) is given by the radius $r$ of this droplet via

$$
m=\frac{4}{3} \pi r^{3} \rho_{l}
$$


where $\rho_{l}$ denotes the (volumetric) density of liquid water. We also employ temperature $T$, pressure $p$, and water vapour concentration $q_{v}$ as thermodynamical variables. To a very good approximation we can assume air (index $a$ ) and water vapour (index $v$ ) as ideal gases, using the ideal gas law

$$
p_{x} V=M_{X} R_{X} T \Longleftrightarrow p_{x}=\rho_{x} R_{X} T
$$

with $M_{x}$ the mass, and $R_{x}=R^{\star} / M_{\text {mol, } x}$ the specific gas constant for the substance $x \in\{a, v\}$, given the universal gas constant $R^{\star}$ and the respective molar mass $M_{\text {mol, } x}$. Generally, Dalton's law is applied, i.e., the total pressure is assumed to satisfy $p=p_{a}+p_{v}$. Since $p_{v} \ll p_{a}$ and $\rho_{v}=q_{v} \rho_{a} \ll \rho_{a}$ we usually use the approximation $p \approx p_{a}$ and $\rho=\rho_{v}+\rho_{a} \approx \rho_{a}$. We thus have

$$
\rho=\frac{p}{R_{a} T}, \quad R_{a}=287.05 \mathrm{~J} \mathrm{~kg}^{-1} \mathrm{~K}^{-1},
$$

to a high level of accuracy.

The thermodynamic equilibrium between water vapour and liquid water is determined by the ClausiusClapeyron equation, describing the saturation vapour pressure $p_{s}(T)$. For the latter we use the approximation provided in Section A due to [31], compare (44). The saturation vapour concentration for water vapour can be approximated by

$$
q_{v s}(p, T) \approx \varepsilon \frac{p_{s}(T)}{p}, \quad \varepsilon=\frac{M_{\mathrm{mol}, v}}{M_{\mathrm{mol}, a}} \approx 0.622 .
$$

The latent heat of the phase transition between vapour and liquid water is set to the constant value $L=$ $2.53 \cdot 10^{6} \mathrm{~J} \mathrm{~kg}^{-1}$.

For our description of warm clouds we make the following assumptions:

1. We distinguish our two liquid species according to their particle sizes: cloud droplets are small with a diameter below $50 \mu \mathrm{m}$, in general, while rain drops are much larger.

2. Since cloud droplets are small, their sedimentation velocity is negligible. Rain drops, on the other hand, are large enough to fall, and they have a terminal fall velocity $v_{t}$ depending on their size, as can be derived from theory and measurements [34]. This distinction has been introduced by [15] for the first time.

3. Only cloud droplets can be formed out of the gas phase, i.e., water droplets grow from activated aerosols due to Köhler theory $[1,19]$.

4. Cloud droplets and rain drops can grow and evaporate due to diffusion of water vapour, but we neglect diffusional growth of rain drops, since it is very slow [3]; this assumption is used in many models [e.g. 21].

5. Rain drops form and grow by collision of/with cloud droplets; this is the major pathway for the growth of large water particles [16].

For the formulation of a corresponding system of equations for the time evolution of the cloud system we thus consider the following processes (see Figure 1):

- Formation of cloud droplets due to condensation (process $C$ ).

- Growth/evaporation of cloud and rain particles due to diffusion (processes $C, E, E^{\prime}$ ).

- Collision of particles for forming rain drops (processes $A_{1}, A_{1}^{\prime}, A_{2}$ ).

- Sedimentation of rain drops (processes $S, S^{\prime}$ ). 


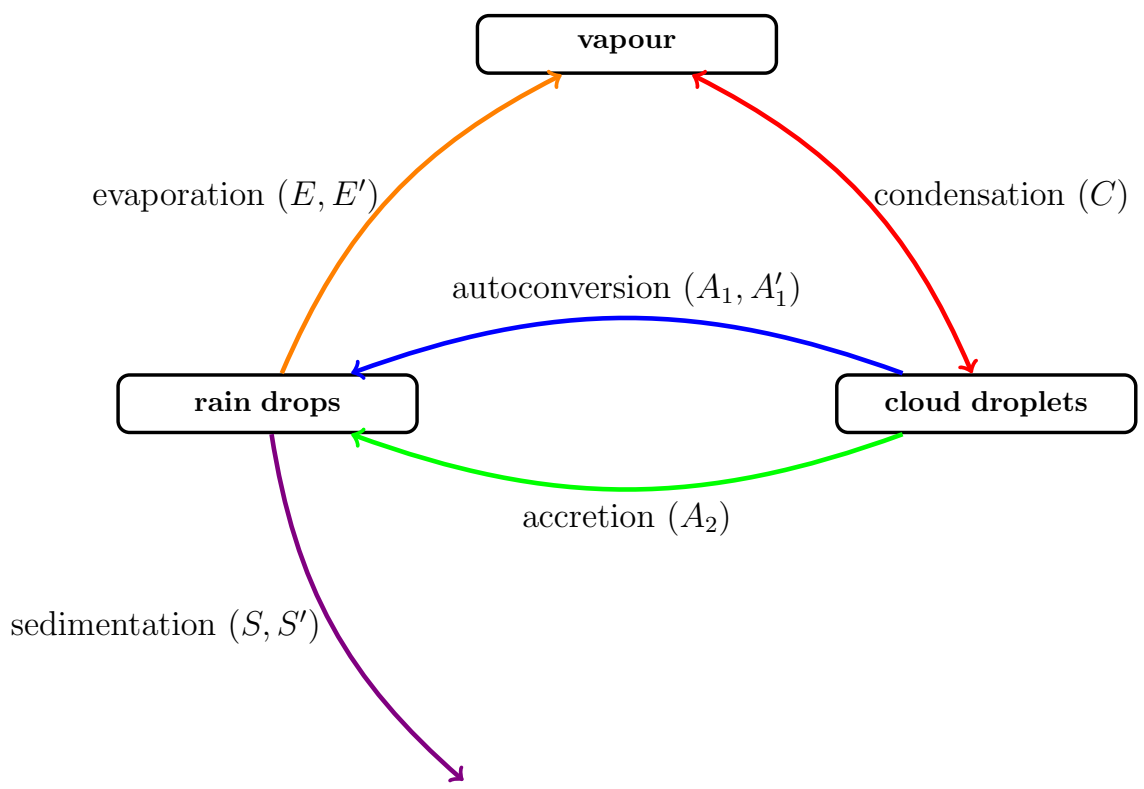

Figure 1: Processes and interactions between the water species (vapour, cloud droplets and rain drops) in the model.

As a general rule we first investigate rates on a single particle basis, if possible. In a second step we extend this ansatz to derive corresponding rates for the bulk variables mass and number concentrations, respectively. This then yields differential equations for the cloud variables $q_{c}, q_{r}, n_{r}$, and the thermodynamic variables $q_{v}, p, T$, which are coupled to additional algebraic state equations for $n_{c}$ and $\rho$ for closing the system.

\subsubsection{Terminal velocity of water particles}

A water droplet of spherical shape is accelerated by gravity. On the other hand, friction of air is changing momentum in the opposite direction. Eventually, the balance of forces leads to a constant velocity of the particle, the so called terminal velocity $v_{t}$. There are different descriptions of $v_{t}$ in the pertinent literature; as our gold standard we quote from [36, their eq. (4)] the formula

$$
v_{t}(r)=\alpha_{r}-\beta_{r} \exp \left(-\gamma_{r} 2 r\right)
$$

with

$$
\alpha_{r}=9.292 \mathrm{~m} \mathrm{~s}^{-1}, \quad \beta_{r}=9.623 \mathrm{~m} \mathrm{~s}^{-1}, \quad \gamma_{r}=6.222 \cdot 10^{2} \mathrm{~m}^{-1}
$$

for larger drops with radius $r>50 \mu \mathrm{m}$. A simpler approximation from [37] uses the power law ansatz

$$
v_{t}(m)=\alpha^{\prime} m^{\beta} \quad \alpha^{\prime}=159 \mathrm{~m} \mathrm{~s}^{-1} \mathrm{~kg}^{-\beta}, \quad \beta=\frac{4}{15},
$$

depending on the drop mass $m$, connected to the radius via eq. (1). Note, that this approximation was formulated originally by [22]. These approximations of $v_{t}$ provide reference values of the terminal velocity, which correspond to the density

$$
\rho=\rho_{\star}=1.225 \mathrm{~kg} \mathrm{~m}^{-3}
$$

of dry air at normal pressure $p_{\star}=101325 \mathrm{~Pa}$ and temperature $T_{\star}=288 \mathrm{~K}$. For other densities they have to be adapted, using the ansatz $\left(\rho_{\star} / \rho\right)^{c}$ as discussed in [32, their Appendix A], with the exponent $c=\frac{1}{2}$ for large rain drops.

Here we propose the functional relation

$$
v_{t}(m)=\alpha m^{\beta}\left(\frac{m_{t}}{m+m_{t}}\right)^{\beta}\left(\frac{\rho_{\star}}{\rho}\right)^{\frac{1}{2}}
$$




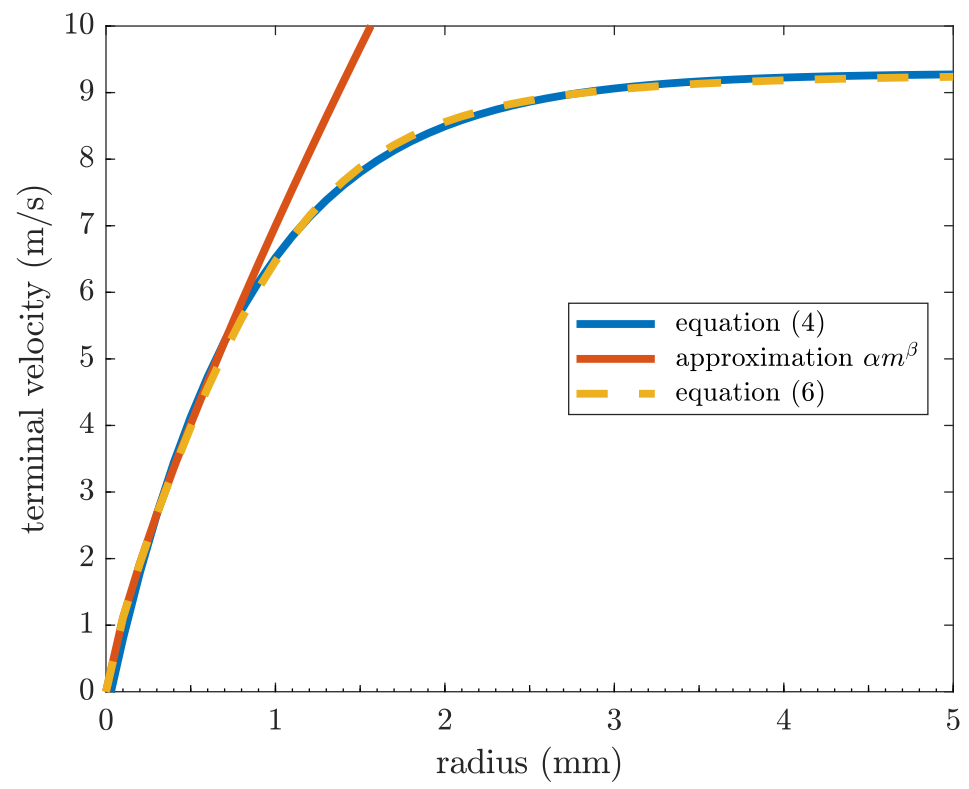

Figure 2: Terminal velocity $v_{t}$ as a function of the radius; values are calculated for the density $\rho=\rho_{\star}$ : Blue: “Gold standard" (4) from [36]; red: simple approximation $v_{t}=\alpha m^{\beta}$ in the spirit of [37]; yellow: new approximation (6).

with $\alpha=190.3 \mathrm{~m} \mathrm{~s}^{-1} \mathrm{~kg}^{-\beta}$ and $m_{t}=1.21 \cdot 10^{-5} \mathrm{~kg}$, which is similar to the [37] model, but shows the same asymptotic behaviour as (4) for large rain drops, compare Figure 2. Using the approximation $m \approx q_{r} / n_{r}$ we thus obtain the formula

$$
v_{t}=\alpha q_{r}^{\beta}\left(\frac{m_{t}}{q_{r}+m_{t} n_{r}}\right)^{\beta}\left(\frac{\rho_{\star}}{\rho}\right)^{\frac{1}{2}},
$$

to be used in our parameterization.

\subsubsection{Diffusional growth/evaporation for a single water particle}

The growth/evaporation of a single water particle of spherical shape with radius $r$ and mass $m$ can be formulated as follows [see, e.g., 34]:

$$
\frac{\mathrm{d} m}{\mathrm{~d} t}=-4 \pi \operatorname{Dr} \rho\left(q_{v s}-q_{v}\right) G f_{v},
$$

which involves the following terms:

- A diffusion constant [cf. 34]

$$
D=D_{0}\left(\frac{T}{T_{0}}\right)^{1.94} \frac{p_{\star}}{p}
$$

depending on temperature and pressure with

$$
D_{0}=2.11 \cdot 10^{-5} \mathrm{~m}^{2} \mathrm{~s}^{-1}, \quad T_{0}=273.15 \mathrm{~K} .
$$

- The influence of latent heat release is given by

$$
G=\left[\left(\frac{L}{R_{V} T}-1\right) \frac{L p_{s}}{R_{V} T^{2}} \frac{D}{K}+1\right]^{-1},
$$


where the thermal conductivity $K$ is given ${ }^{1}$ by [4]

$$
K(T)=\frac{a_{K} T^{\frac{3}{2}}}{T+b_{K} 10^{\frac{c_{K}}{T}}}
$$

with

$$
a_{K}=0.002646 \mathrm{~W} \mathrm{~m}^{-1} \mathrm{~K}^{-1} \mathrm{~K}^{-\frac{3}{2}}, \quad b_{K}=245.4 \mathrm{~K}, \quad c_{K}=-12 \mathrm{~K} .
$$

- A correction for ventilation effects: If a large particle, i.e., a rain drop, is falling through air, vortices and turbulence are induced, which enhance evaporation [35]. To account for this, an additional empirical ventilation coefficient $f_{v}$ is introduced in (8); according to [37] we let

$$
f_{v}=a_{v}+b_{v} N_{\mathrm{Sc}}^{\frac{1}{3}} N_{\mathrm{Re}}^{\frac{1}{2}}, \quad a_{v}=0.78, \quad b_{v}=0.308,
$$

where the Schmidt and Reynolds numbers are defined as

$$
N_{\mathrm{Sc}}=\frac{\mu}{\rho D}, \quad N_{\mathrm{Re}}=\frac{2 \rho r}{\mu} v_{t}=\frac{2 \rho}{\mu} v_{t}\left(\frac{3}{4 \pi}\right)^{\frac{1}{3}} \rho_{l}^{\frac{1}{3}} m^{\frac{1}{3}},
$$

in terms of the dynamic viscosity $\mu$ of air. The latter can be expressed as a function of temperature [cf. 4], i.e.,

$$
\mu=\frac{\mu_{0} T^{\frac{3}{2}}}{T+T_{\mu}}, \quad \mu_{0}=1.458 \cdot 10^{-6} \mathrm{sPaK}^{-\frac{1}{2}}, \quad T_{\mu}=110.4 \mathrm{~K} .
$$

For cloud droplets we can neglect ventilation effects, hence the mass rate of a cloud droplet is given by

$$
\frac{\mathrm{d} m_{c}}{\mathrm{~d} t}=-4 \pi D r \rho\left(q_{v s}-q_{v}\right) G=d \rho\left(q_{v}-q_{v s}\right) m_{c}^{\frac{1}{3}}
$$

with

$$
d=4 \pi\left(\frac{3}{4 \pi \rho_{l}}\right)^{\frac{1}{3}} D G .
$$

On the other hand, for rain drops we neglect condensation, hence

$$
\frac{\mathrm{d} m_{r}}{\mathrm{~d} t}=-d \rho\left(q_{v s}-q_{v}\right)_{+}\left[a_{E} m_{r}^{\frac{1}{3}}+b_{E} v_{t}\left(m_{r}\right)^{\frac{1}{2}} m_{r}^{\frac{1}{2}}\right],
$$

where

$$
a_{E}=a_{v}=0.78, \quad b_{E}=b_{v}\left(\frac{\mu}{\rho D}\right)^{\frac{1}{3}} \sqrt{\frac{2 \rho}{\mu}}\left(\frac{3}{4 \pi \rho_{l}}\right)^{\frac{1}{6}} .
$$

Here we have used the short-hand notation

$$
\left(q_{v s}-q_{v}\right)_{+}= \begin{cases}q_{v s}-q_{v}, & q_{v} \leq q_{v s} \text { (subsaturated regime) } \\ 0, & q_{v}>q_{v s} \text { (supersaturated regime) } .\end{cases}
$$

Remark 2.1. A more general approach suggested, e.g., in [34] also includes a kinetic correction of the diffusivity $D$ for very small droplets. Since calculations show that these corrections are not relevant for cloud droplets with a radius $r>5 \mu \mathrm{m}$ we omit these corrections in this study.

1 We have corrected a typo in the original formulation by [4], after comparison with tabulated values of $K$, i.e., we have multiplied $a_{K}$ by a factor of 0.1 . 


\subsubsection{Collision of rain drops with cloud droplets and coalescence/accretion}

A spherical rain drop of radius $r$ and mass $m_{r}$ falls with terminal velocity $v_{t}\left(m_{r}\right)$ through a cylindrical volume

$$
V=\pi r^{2} v_{t}\left(m_{r}\right) \Delta t=\pi\left(\frac{3}{4 \pi \rho_{l}}\right)^{\frac{2}{3}} m_{r}^{\frac{2}{3}} v_{t}\left(m_{r}\right) \Delta t
$$

during a time interval $\Delta t>0$. Within this volume there is a total mass $M_{c}=V \rho q_{c}$ of cloud droplets that will be hit by this rain drop. The corresponding mass growth rate of the rain drop is thus given by

$$
\frac{\mathrm{d} m_{r}}{\mathrm{~d} t}=k_{2}^{\prime} V \rho q_{c}=k_{2}^{\prime} \rho q_{c} \pi\left(\frac{3}{4 \pi \rho_{l}}\right)^{\frac{2}{3}} m_{r}^{\frac{2}{3}} v_{t}\left(m_{r}\right),
$$

where $k_{2}^{\prime}>0$ is the associated efficiency parameter.

\subsection{Computing mass/number concentration rates from single particles rates}

In this section we derive rates of change of the bulk quantities. For this purpose rates for single particles are scaled up with the corresponding particle number concentration.

\subsubsection{Rates for diffusional growth/evaporation of rain drops}

Evaporation of rain drops affects mass concentration but also number concentration, because small droplets may evaporate completely. We therefore use

$$
E=-\left.n_{r} \frac{\mathrm{d} m_{r}}{\mathrm{~d} t}\right|_{\text {evaporation }}
$$

for the evaporation term of the mass concentration rate, and we assume that the reduction of the number concentration is proportional to the evaporated mass; the proportionality factor is set to be equal to the inverse of the average mass $\bar{m}_{r}$ of rain drops. Accordingly, we let

$$
E^{\prime}=\frac{1}{\bar{m}_{r}} E
$$

be the corresponding number concentration rate. Inserting (11) we thus obtain

$$
E=d \rho\left(q_{v s}-q_{v}\right)_{+}\left(a_{E} q_{r}^{\frac{1}{3}} n_{r}^{\frac{2}{3}}+b_{E} v_{t}^{\frac{1}{2}} q_{r}^{\frac{1}{2}} n_{r}^{\frac{1}{2}}\right)
$$

and

$$
E^{\prime}=\frac{1}{\bar{m}_{r}} d \rho\left(q_{v s}-q_{v}\right)_{+}\left(a_{E} q_{r}^{\frac{1}{3}} n_{r}^{\frac{2}{3}}+b_{E} v_{t}^{\frac{1}{2}} q_{r}^{\frac{1}{2}} n_{r}^{\frac{1}{2}}\right)
$$

where $v_{t}$ is given by (7).

\subsubsection{Rates for the accretion of rain drops}

Concerning the collision processes between rain drops and cloud droplets we obtain in the same way the corresponding mass concentration rate

$$
\begin{aligned}
A_{2} & =\left.n_{r} \frac{\mathrm{d} m_{r}}{\mathrm{~d} t}\right|_{\text {accretion }}=n_{r} k_{2} \rho q_{c} \pi\left(\frac{3}{4 \pi \rho_{l}}\right)^{\frac{2}{3}} m_{r}^{\frac{2}{3}} v_{t} \\
& =k_{2} \pi\left(\frac{3}{4 \pi \rho_{l}}\right)^{\frac{2}{3}} v_{t} \rho q_{c} q_{r}^{\frac{2}{3}} n_{r}^{\frac{1}{3}},
\end{aligned}
$$


where we have approximated $m_{r} \approx q_{r} / n_{r}$ in the last step. Note that this is a simplistic result which does not take into account, for example, that the average velocity of rain drops is not equal to the terminal velocity of a rain drop with average mass; but this can be compensated by calibrating the efficiency parameter $k_{2}$, which may be somewhat different from $k_{2}^{\prime}$ in (13).

Remark 2.2. For smaller rain drops one can approximate $v_{t} \approx \alpha\left(q_{r} / n_{r}\right)^{\beta}=\alpha q_{r}^{\beta} n_{r}^{-\beta}$ with $\beta=4 / 15$, cf. (5), and then

$$
A_{2} \sim q_{c} q_{r}^{\frac{2}{3}+\beta} n_{r}^{\frac{1}{3}-\beta}=q_{c} q_{r}^{\frac{14}{15}} n_{r}^{\frac{1}{15}} .
$$

This is almost equivalent to a standard predator-prey formulation $A_{2} \sim q_{c} q_{r}$ with rain drops as predator population, depleting the prey population of cloud droplets [see, e.g., 38].

\subsubsection{Sedimentation of rain drops}

So far all the considered processes take place within each individual control volume. Sedimentation, on the other hand, produces a flux through the boundaries of the control volumes. Let $z$ be the coordinate of the vertical position (above sea level) of the control volume. As stated above rain drops accelerate due to gravity to a reasonable terminal velocity. This will be used to derive fluxes for the bulk variables mass and number concentrations of the rain drops to specify their vertical advection. Distinguishing between effective velocities $v_{q}$ and $v_{n}$ for mass and number concentrations, respectively, the corresponding fluxes $J$ and $J^{\prime}$ are given by

$$
J=v_{q} \rho q_{r}, \quad J^{\prime}=v_{n} \rho n_{r}
$$

with units

$$
[J]=\mathrm{kg} \mathrm{m}^{-2} \mathrm{~s}^{-1}, \quad\left[J^{\prime}\right]=\mathrm{m}^{-2} \mathrm{~s}^{-1} .
$$

The effective velocities $v_{q}$ and $v_{n}$ correlate with the terminal velocity $v_{t}$ of a single drop with average mass, i.e., we let

$$
v_{q}=c_{q} v_{t}, \quad v_{n}=c_{n} v_{t}
$$

with parameters

$$
c_{q}>c_{n}>0 \text {. }
$$

The weight $c_{q}$ takes into account that the size distribution of rain drops is often observed to have heavy tails [26], and that larger drops contribute more to the mass sedimentation flux than smaller ones. In contrast, drops smaller than the mean size yield the dominant contribution to the sedimentation number flux. In short, one can say that more larger drops than smaller ones fall out of a box, and this is taken into account by our constraints (18) on the parameters $c_{q}$ and $c_{n}$ [see, e.g., the discussion in 39].

Remark 2.3. The condition (18) is fulfilled in a natural way when using the exponential or the Gamma distribution for the mass distribution of rain drops [as suggested, e.g., in 37], because this is equivalent to the inequality of moments $\mu_{\beta+1} \mu_{0} \geq \mu_{\beta} \mu_{1}$ of the distributions with $\beta \in \mathbb{R}_{+}$; this is true, since $\log \mu_{r}$ fullfills Lyapunov's inequality [25].

In the column model the sedimentation terms

$$
S=\frac{1}{\rho} \frac{\partial J}{\partial z}, \quad S^{\prime}=\frac{1}{\rho} \frac{\partial J^{\prime}}{\partial z}
$$

appear as sources and sinks, respectively, in the time evolution, and turn the overall model into a hyperbolic system of partial differential equations. For the box model without flux from above, we can simplify the sedimentation terms $S$ and $S^{\prime}$ by using the vertical extension $h$ of the box to obtain

$$
S=S_{\text {out }}=\frac{J}{h \rho}=\frac{v_{q} q_{r}}{h}, \quad S^{\prime}=S_{\text {out }}^{\prime}=\frac{J^{\prime}}{h \rho}=\frac{v_{n} n_{r}}{h} .
$$

Note, that the height of the box may change with time due to adiabatic expansion or compression. See also the discussion in Section 2.6. 


\subsection{Collision of cloud droplets - autoconversion}

In a control volume $\Delta V$ the volume fraction occupied by cloud droplets is given by $\rho q_{c} / \rho_{l}$. The probability that any single cloud droplet collides with any other cloud droplet and that they recombine to a rain drop called autoconversion - is proportional to the size of this volume fraction. It follows that

$$
k_{1} \frac{\rho q_{c}}{\rho_{l}} \Delta t
$$

is the expected number of autoconversions of an individual cloud droplet in a sufficiently small time interval $\Delta t$, where $k_{1}$ with $\left[k_{1}\right]=\mathrm{s}^{-1}$ is the corresponding proportionality constant. Note that we are only interested in those collisions, which result in a single drop which is large enough to be registered as a new rain drop, because the other collisions have no effect on our concentration variables. In addition, the effect of such collisions is quite small.

Multiplying eq. (20) with the total number of cloud droplets $\rho n_{c} \Delta V$ in the control volume we get the number of autoconversions

$$
\frac{1}{2} k_{1}\left(\rho n_{c}\right) \frac{\rho q_{c}}{\rho_{l}} \Delta t \Delta V
$$

in $\Delta V$ within the time interval $\Delta t$; the factor $\frac{1}{2}$ prevents double counting of events.

Since each autoconversion recombines two cloud droplets into a new rain drop, the corresponding rate of the number concentration of rain drops per mass of dry air (i.e., $\rho \Delta V$ ) is given by

$$
A_{1}^{\prime}=k_{1} \frac{\rho n_{c} q_{c}}{2 \rho_{l}} \text {. }
$$

On the other hand, each collision increments the mass of rain drops by two times the average mass $\bar{m}_{c}=q_{c} / n_{c}$ of cloud droplets, which leads to the autoconversion rate for the rain drop mass concentration

$$
A_{1}=2 k_{1} \bar{m}_{c} \frac{\rho n_{c} q_{c}}{2 \rho_{l}}=k_{1} \frac{\rho q_{c}^{2}}{\rho_{l}} .
$$

\subsection{Treatment of cloud droplet condensation}

The treatment of cloud droplet condensation leads to several subtle issues, which must be considered carefully in the development of a consistent and numerically tractable scheme.

\subsubsection{Particle formation}

In the atmosphere many aerosol particles are available. Some of them, depending on their chemical components (i.e., their hygroscopicity), have the ability to attract water molecules. As soon as there is water vapour these particles grow by diffusion, i.e., a phase transition from the gas phase to mixed particles including liquid water takes place. This effect can be described by Köhler theory [see, e.g., 19, 34], which determines the size of the grown aerosol at a given saturation ratio $q_{v} / q_{v s}$ in dependence of the initial size of the aerosol and its chemical properties. A more compact formulation of this theory can be found in [33], using the hygroscopicity as single parameter. The Köhler theory predicts a so-called critical radius $r_{0}$ and a maximal supersaturation ratio $S_{0}=q_{v} / q_{v s}>1$, such that there is a one-to-one relation between $q_{v}<S_{0} q_{v s}$ and the radius $0<r<r_{0}$ of a given wetted aerosol. Once the saturation has reached the critical level $S_{0}$ the aerosol particle becomes unstable, i.e., it can grow to (almost) arbitrarily large sizes; this grown aerosol particle can now be called a cloud droplet.

There are complex cloud models, which try to take into account the complicated procedure of activation, but most standard cloud models do not consider aerosol particles, so that the generation or activation of droplets must be treated in a somewhat artifical manner. Often, for example, a certain number of cloud droplets is activated, once a certain threshold of supersaturation is reached. In even simpler models (mostly 


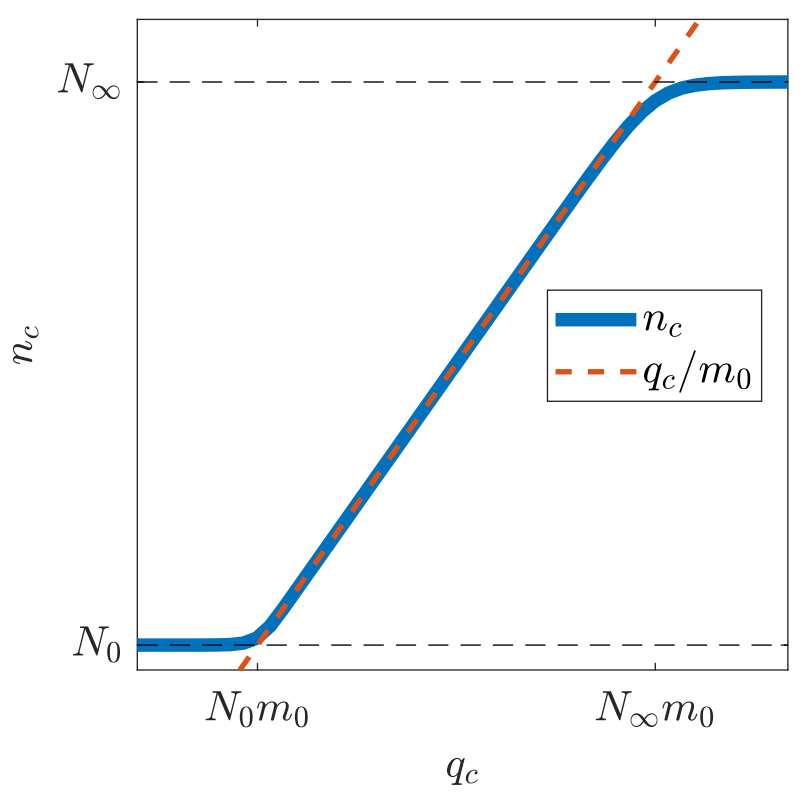

Figure 3: Number concentration $n_{c}$ depending on the mass concentration $q_{c}$ as given in equation (22).

single moment schemes), it is often assumed that in case of supersaturation all aerosol particles are activated instantaneously, i.e., $n_{c}$ is set to the number concentration of available CCN [see, e.g., 11]; likewise the number concentration is set to zero in subsaturated conditions.

In our model we assume that there are $N_{0}$ aerosols (per volume and mass of dry air) which reach the critical Köhler radius first, e.g., because they are largest. This implies that the counting concentration $n_{c}$ is already positive before the first droplets can be registered, i.e., when $q_{c}$ becomes positive. Later, other aerosols may turn into droplets, and we further assume, that there are at most $N_{\infty}$ aerosols (per volume and mass of dry air) available. To be specific, we assume that $n_{c}$ is a function of $q_{c}$, namely

$$
n_{c}=\frac{q_{c} N_{\infty}}{q_{c}+N_{\infty} m_{0}} \operatorname{coth}\left(\frac{q_{c}}{N_{0} m_{0}}\right)
$$

with free parameters $N_{\infty}, N_{0}, m_{0}$, see Figure 3.

This function represents three different regimes: (i) Before the maximal saturation level $S_{0}$ is reached, small aerosol particles are already around, but the total liquid droplet mass concentration is still negligible (i.e., $q_{c}=0$ ). However, the number concentration is already equal to the parameter $N_{0}$. (ii) At growing supersaturation, more and more cloud droplets appear, and all aerosols compete for the available water vapour, so that the mean size of all particles is approximately constant. Therefore, in this regime there is an approximately linear relation between $n_{c}$ and $q_{c}$, i.e., $q_{c} \approx m_{0} n_{c}$. In particular, this implies that we have an ongoing in-cloud activation of new cloud droplets with increasing saturation rate. The parameter $m_{0}$ can be interpreted as the typical water mass content of a cloud droplet close to activation. (iii) At high supersaturation levels all CCN are activated, thus the droplet number concentration is almost equal to the total number of CCNs, i.e., $n_{c} \approx N_{\infty}$.

We will demonstrate in Section 3.4 below that this nonlinear coupling of the droplet number and mass concentrations entails an automatic (i.e., implicit) particle activation. By changing the tuning parameters $N_{0}, N_{\infty}$, and $m_{0}$ it is possible to represent different aerosol regimes (i.e. polluted, clean, maritime regimes; compare Section 4.3).

Remark 2.4. We found that the algebraic constraint (22) is better suited for modelling the activation of cloud particles on physical grounds than any of the differential equations for $n_{c}$ as a function of time that we could think of. 


\subsubsection{Condensation rate for cloud droplets}

In warm clouds the amount of available water molecules in the gas phase is very high and the diffusivity is quite large, hence diffusional growth of droplets is a very fast process, if cloud droplets are already available. Therefore supersaturation due to cooling of air, for example, changes very rapidly towards thermodynamic equilibrium, i.e., $q_{v} \approx q_{v s}$. Accordingly, many cloud models use saturation adjustment, which means that for $q_{v}>q_{v s}$ all excess water vapour is instantaneously turned into cloud droplet mass concentration $q_{c}$, so that $q_{v}=q_{v s}$.

Saturation adjustment can be solved numerically in a very efficient way by using Newton's method [18]. However, the method leads to some problematic phenomena. First, as we have discussed in Section 2.4.1, the activation of droplets inside clouds is nonphysical, strictly speaking, since this activation requires supersaturation. Therefore activation of cloud droplets has to be carried out separately before saturation adjustment is performed. Second, saturation adjustment has been shown to lead to an overestimation of latent heat release during condensation, because all excess water vapour is turned into liquid water at once. This yields higher buoyancy and introduces errors in the representation of systems with convective updrafts [see, e.g., 7, 9, 10]. Therefore saturation adjustment should be avoided whenever possible, and explicit supersaturation regimes should be tolerated in modern cloud models; see also Section 4.2.

As we have explained before, our model does allow supersaturation, and the growth rate of the mass concentration $q_{c}$ follows from the growth equation (9) to be

$$
C=n_{c} \frac{\mathrm{d} m_{c}}{\mathrm{~d} t}=d \rho\left(q_{v}-q_{v s}\right) n_{c}^{\frac{2}{3}} q_{c}^{\frac{1}{3}},
$$

where we have taken $m_{c}=q_{c} / n_{c}$ to be the average mass of a cloud droplet.

\subsection{Full model equations: Box model}

The cloud model variables have to be coupled to thermodynamics, i.e., to changes in pressure and temperature, respectively. For this we assume adiabatic changes (no heat exchange with the environment) when the control volume is moving vertically. The adiabatic lapse rate $\gamma=g / c_{p}$ is used for these temperature changes. The latent heat of a phase transition (water vapour $\leftrightarrow$ liquid water) is also distributed in the volume, changing temperature. In addition, we assume hydrostatic pressure change $\left(\frac{\partial p}{\partial z}=-g \rho\right)$, which is a common assumption [e.g., 20].

Now we can formulate the system of equations for a box model approach, given the different sinks and sources of the water quantitities as described above. We also assume some external forcing in terms of a (given) vertical upward motion with velocity $w=w(t)$; the latter can be used to model, e.g., the passage over a mountain ridge or the ascent of a warm front onto cold air; see Section 4.4 for examples. The system is given by

$$
\begin{aligned}
& \dot{q}_{v}=-C \quad+E, \\
& \dot{q}_{c}=C-A_{1}-A_{2} \text {, } \\
& \dot{q}_{r}=\quad A_{1}+A_{2}-E-S \text {, } \\
& \dot{n}_{r}=A_{1}^{\prime} \quad-E^{\prime}-S^{\prime} \text {, } \\
& \dot{p}=-\gamma \rho w \text {, } \\
& \dot{T}=-\gamma w-\frac{L}{c_{p}}(E-C),
\end{aligned}
$$

compare Figure 1. Its right-hand side depends on intermediate quantities, but also on the coupled number concentration $n_{c}$ of cloud droplets and on the density $\rho$ of dry air; therefore, the system is closed using the corresponding algebraic constraints

$$
\begin{aligned}
n_{c} & =\frac{q_{c} N_{\infty}}{q_{c}+N_{\infty} m_{0}} \operatorname{coth}\left(\frac{q_{c}}{N_{0} m_{0}}\right), \\
\rho & =\frac{p}{R_{a} T} .
\end{aligned}
$$




\subsection{Solvability of the differential equations}

Since the right-hand side of the differential equation (24) is not differentiable, no higher order regularity of the solution can be expected. Moreover, the Picard-Lindelöf theory is not applicable, so that the differential equation has no unique solution, in general. The existence of solutions is nevertheless guaranteed by Peano's theory [40].

The lack of uniqueness is apparent from the differential equation for $q_{c}$ : If the system is in the subsaturated regime, i.e., if $q_{c}, n_{r}$, and $q_{r}$ are zero at time $t=0$, then all the driving terms $A_{1}, A_{1}^{\prime}, A_{2}, C, E, E^{\prime}, S$, and $S^{\prime}$ on the right-hand side of (24) vanish, and hence, the constant functions $q_{v}=q_{v}(0), q_{c}=0, q_{r}=0$, and $n_{r}=0$, solve the first four differential equations in (24) - even if the system changes to the supersaturated regime at some later time $t=t_{0}$ for a specific choice of upward drift $w$. However, as will be shown in Section 3.4, as soon as $q_{v s}<q_{v}$ our specific ansatz for generating cloud droplets allows for a nontrivial solution of the system (24).

In our box model we treat the total mass $m_{a}$ of dry air within the box as being constant over time, and we also freeze the horizontal cross section $A$ of the box. According to the gas law (2), however, the density $\rho$ may vary with time. We therefore need to adapt the vertical extent $h=h(t)$ of the box to account for changes in the density and to conserve the total air mass $m_{a}=\rho A h$ over time.

\subsection{Formulation of a mass conserving column model}

The model of Section 2.5 can readily be extended to a vertical column of air in order to treat nontrivial vertical humidity distributions. To this end multiple boxes are stacked on top of each other. Strictly speaking, the column model consists of an initial value problem for a hyperbolic differential algebraic system, where we consider a Lagrangian air column with internal sedimentation flow. The individual boxes provide a natural finite volume discretization in space; the time discretization will be worked out in more detail in Section 3.

Concerning the conservation of mass (of dry air and of water, respectively) we assume that the horizontal cross section of all boxes is the same and that its area is $A$, and as for the box model we adapt the height $h=h(t, z)$ of each individual box over time to conserve the mass $m_{a}=m_{a}(z)$ of dry air within every individual box; the mass may, however, depend on the spatial variable $z$, i.e., be different for each box. As we will see in Section 3.2 this way we not only conserve the mass of air, but also the total mass of water within the column - except for precipitation, of course.

\section{Numerical time integration}

Starting from (consistent) initial values for the variables of our model at time $t=0$, the overall column model system with a given forcing velocity profile $w=w(t)$ can be integrated by stepping forward explicitly in time. For the hyperbolic column model this calls for a Courant-Friedrichs-Lewy (CFL) condition, i.e., an upper bound of the time step $\tau>0$. In our context this constraint has an obvious physical interpretation: The inflow of falling rain drops (both, in terms of mass and number) into any given box must not traverse this box within a single time step, so that the flows across all horizontal box faces are independent of each other for every fixed time step. Because of our assumption $v_{q}>v_{n}$, see (17), (18), this amounts to the upper bound

$$
\tau<\min \left\{h / v_{q}\right\}, \quad v_{q}=c_{q} v_{t},
$$

the minimum being taken over all boxes at a given time step.

In order to maintain nonnegativity of all water concentrations, see Sections 3.3 and 3.4 for more details, we split the sedimentation term into its outflow and inflow components, $S_{\text {out }}$ and $S_{\text {in }}$, respectively, and treat them separately as sinks and sources. In this manner the resulting overall system can be considered an ordinary differential algebraic system. For the numerical treatment it is important that this differential algebraic 
system has index one, i.e., that the closing conditions (26) and (25) can be solved (explicitly) for the algebraic variables $\rho$ and $n_{c}$. By updating these algebraic variables in each time step after all other variables - except for $h$, see Section 3.2 below - we make sure that the two algebraic constraints are consistent after each individual time step.

Concerning the other variables we use a semi-implicit Euler scheme, as worked out in detail in the following sections, where certain variables are treated explicitly, while other variables are solved for implicitly, but very efficiently. At the beginning of each time step we evaluate all the parameters of the different processes, i.e., the saturation vapour concentration (3), the terminal velocity (6), the diffusivity (10), and the ventilation parameter $b_{E}$ of (12), using the values of the depending variables from the previous time step.

\subsection{The semi-implicit Euler scheme}

We split the right-hand side of the differential equation (24) into two parts, one of which is being treated implicitly, while the other one is treated explicitly. For both parts we use the Euler scheme, because the solution lacks regularity in general; see Section 2.6. The implicit part contains the entire right-hand side for the cloud droplet mass concentration and includes all the sinks for the rain drop mass and number concentrations. This splitting ensures:

(a) an adequate activation of cloud droplets as soon as the system becomes supersaturated, and

(b) that all water concentrations remain nonnegative.

To be specific, starting from the current values $q_{v, i}, q_{c, i}, q_{r, i}, n_{r, i}, n_{c, i}, p_{i}, T_{i}$, and $\rho_{i}$ of the approximate solution of (24)-(26) in some given box at time $t_{i}=i \tau$, we first solve

$$
\begin{aligned}
& q_{r, i+1 / 2}=q_{r, i}-\tau\left(E\left(q_{r, i+1 / 2}, n_{r, i+1 / 2}\right)+S_{\text {out }}\left(q_{r, i+1 / 2}\right)\right), \\
& n_{r, i+1 / 2}=n_{r, i}-\tau\left(E^{\prime}\left(q_{r, i+1 / 2}, n_{r, i+1 / 2}\right)+S_{\text {out }}^{\prime}\left(n_{r, i+1 / 2}\right)\right),
\end{aligned}
$$

for $q_{r, i+1 / 2}$ and $n_{r, i+1 / 2}$. Then we determine $q_{c, i+1}$ from

$$
q_{c, i+1}=q_{c, i}+\tau\left(C\left(q_{c, i+1}\right)-A_{1}\left(q_{c, i+1}\right)-A_{2}\left(q_{c, i+1}, q_{r, i+1 / 2}, n_{r, i+1 / 2}\right)\right),
$$

and finally, we update the new values of $q_{r}$ and $n_{r}$ as

$$
\begin{aligned}
& q_{r, i+1}=q_{r, i+1 / 2}+\tau\left(A_{1}\left(q_{c, i+1}\right)+A_{2}\left(q_{c, i+1}, q_{r, i+1 / 2}, n_{r, i+1 / 2}\right)+S_{\mathrm{in}}\right), \\
& n_{r, i+1}=n_{r, i+1 / 2}+\tau\left(A_{1}^{\prime}\left(q_{c, i+1}\right)+S_{\mathrm{in}}^{\prime}\right) .
\end{aligned}
$$

We use the old values $q_{v, i}, n_{c, i}, \rho_{i}, p_{i}$, and $T_{i}$, when evaluating the respective terms on the right-hand sides of (27)-(31). In (30) and (31) the inflows $S_{\text {in }}$ and $S_{\text {in }}^{\prime}$ are given by the corresponding outflows of the neighboring box, which have been determined in steps (27) and (28). This implies that in the implementation of the column model each update (27)-(31) should be done simultaneously for all individual boxes to have the inflows available when needed; note that this allows for a straightforward SIMD parallelization (single instruction, multiple data) of the column model.

In Section 3.3 we show that the system (27), (28) has a unique nonnegative solution $q_{r, i+1 / 2}, n_{r, i+1 / 2}$, which can be written down explicitly. Step (29), on the other hand, is treated in Section 3.4: It can be reduced to the computation of a specific root of a polynomial of degree six, and a straightforward implementation of the Newton method provides a very efficient scheme for the computation of $q_{c, i+1}$, with guaranteed quadratic convergence.

Remark 3.1. In the important special case, where a cloud parcel becomes supersaturated, but no cloud droplets do yet exist, the corresponding solution $q_{c, i+1}$ will be positive; compare item (a) in Section 3.4. Therefore the implicit Euler step (29) is our key means to automatically invoke the activation of cloud droplets discussed in Section 2.4.1. 
Once the new values for $q_{c}, q_{r}$, and $n_{r}$ have been computed, the pressure $p$, the temperature $T$, and the vapour concentration $q_{v}$ are updated with an explicit Euler step, using the quantities

$$
E=E\left(q_{r, i+1 / 2}, n_{r, i+1 / 2}\right) \quad \text { and } \quad C=C\left(q_{c, i+1}\right)
$$

determined in (27) and (29), respectively. Finally the algebraic variables $\rho$ and $n_{c}$ are updated, and the new box heights are retrieved from the identity

$$
h_{i+1}=\frac{m_{a}}{\rho_{i+1} A},
$$

where the mass $m_{a}$ and the area $A$ of the horizontal cross section of each box stay constant over all times. This ensures conservation of the mass of dry air.

\subsection{Water mass conservation}

Assuming that there is no inflow into the column (box) from above, it is obvious from (24) and (32) that the overall water mass balance in the column (box) model is given by

$$
\sum_{\text {boxes }} m_{a}\left(q_{v}+q_{c}+q_{r}\right)=M_{a}-R
$$

where $M_{a}$ is the total mass of water within the column (box) at time $t=0$, and $R$ is the integrated precipitation rate on the ground since $t=0$. Since our semi-implicit Euler scheme is using in every individual box the same values $A_{1}, A_{2}, C$, and $E$ in the different equations and the same inflow and outflow for neighboring boxes, the above identity is also maintained for our discrete time evolution, with $R$ being the accumulated sum of $\tau m_{a} S_{\text {out }}$ of the lowermost box.

\subsection{Numerical solution of the system (27), (28)}

Solving for $E$ in (27) and inserting the corresponding expression into (14) it follows from (28) that

$$
\begin{aligned}
n_{r, i+1 / 2} & =n_{r, i}-\frac{1}{\bar{m}_{r}}\left(q_{r, i}-q_{r, i+1 / 2}-\tau S_{\text {out }, i+1}\left(q_{r, i+1 / 2}\right)\right)-\tau S_{\text {out }, i+1}^{\prime}\left(n_{r, i+1 / 2}\right) \\
& =n_{r, i}-\frac{1}{\overline{m_{r}}}\left(q_{r, i}-q_{r, i+1 / 2}-\tau s q_{r, i+1 / 2}\right)-\tau s^{\prime} n_{r, i+1 / 2}
\end{aligned}
$$

with

$$
s=\frac{v_{q}}{h_{i}}, \quad s^{\prime}=\frac{v_{n}}{h_{i}},
$$

cf. (19). If we take the average mass of rain drops to be

$$
\bar{m}_{r}=q_{r, i} / n_{r, i},
$$

then it follows that $q_{r, i+1 / 2}$ and $n_{r, i+1 / 2}$ are linearly coupled, namely

$$
q_{r, i+1 / 2}=\frac{q_{r, i}}{n_{r, i}} \frac{1+\tau s^{\prime}}{1+\tau s} n_{r, i+1 / 2} .
$$

Inserting this into the definition (14) of $E^{\prime}$ we obtain

$$
\begin{aligned}
E^{\prime} & =\frac{n_{r, i}}{q_{r, i}} d \rho\left(q_{v}-q_{v s}\right)_{+}\left(a_{E} q_{r, i+1 / 2}^{\frac{1}{3}} n_{r, i+1 / 2}^{\frac{2}{3}}+b_{E} q_{r, i+1 / 2}^{\frac{1}{2}} n_{r, i+1 / 2}^{\frac{1}{2}}\right) \\
& =\lambda n_{r, i+1 / 2}
\end{aligned}
$$

for some (computable) $\lambda>0$. It therefore follows from (28) that $n_{r, i+1 / 2}$ satisfies

$$
n_{r, i+1 / 2}=n_{r, i}-\tau\left(\lambda n_{r, i+1 / 2}+s^{\prime} n_{r, i+1 / 2}\right),
$$


i.e.,

$$
n_{r, i+1 / 2}=\frac{1}{1+\tau \lambda+\tau s^{\prime}} n_{r, i}
$$

Inserting (35) into (34) we finally obtain

$$
q_{r, i+1 / 2}=\frac{1+\tau s^{\prime}}{1+\tau s} \frac{1}{1+\tau \lambda+\tau s^{\prime}} q_{r, i} .
$$

Hence, (35) and (36) are the explicit solutions of (27), (28). Note that $q_{r, i+1 / 2}$ and $n_{r, i+1 / 2}$ remain positive, if $n_{r, i}$ and $q_{r, i}$ have been positive; see Section 3.5 for the case when one of the two quantities happens to be zero.

\subsection{Numerical solution of equation (29)}

Given $q_{r, i+1 / 2}$ and $n_{r, i+1 / 2}$, the nonlinear equation (29) for $q_{c, i+1}$ can be rewritten in the form

$$
q_{c, i+1}=q_{c, i}+\tau\left(c q_{c, i+1}^{\frac{1}{3}}-a_{1} q_{c, i+1}^{2}-a_{2} q_{c, i+1}\right)
$$

with

$$
c=d \rho\left(q_{v}-q_{v s}\right) n_{c, i}^{\frac{2}{3}}, \quad a_{1}=k_{1} \frac{\rho}{\rho_{l}}, \quad a_{2}=k_{2} \pi\left(\frac{3}{4 \pi \rho_{l}}\right)^{\frac{2}{3}} v_{t} \rho q_{r, i+1 / 2}^{\frac{2}{3}} n_{r, i+1 / 2}^{\frac{1}{3}},
$$

cf. (23), (21), and (16). While $a_{1}$ and $a_{2}$ are always nonnegative, the sign of $c$ depends on the saturation regime: $c$ is positive in supersaturated regimes, and nonpositive else.

It follows that the nonnegative value of $x=q_{c, i+1}^{\frac{1}{3}}$ is a root of the sixth order polynomial

$$
p(x)=\tau a_{1} x^{6}+\left(1+\tau a_{2}\right) x^{3}-\tau c x-q_{\mathrm{c}, i} .
$$

The first two derivatives of $p$ are given by

$$
\begin{aligned}
p^{\prime}(x) & =6 \tau a_{1} x^{5}+3\left(1+\tau a_{2}\right) x^{2}-\tau c, \\
p^{\prime \prime}(x) & =30 \tau a_{1} x^{4}+6\left(1+\tau a_{2}\right) x,
\end{aligned}
$$

and since $p^{\prime \prime}(x)>0$ for $x>0$ it follows that the graph of $p$ is strictly convex for $x \geq 0$. Moreover, $p(0) \leq 0$, and $p(x) \rightarrow+\infty$ as $x \rightarrow+\infty$.

Now we need to distinguish the following cases (see Figure 4):

(a) In the supersaturated regime we have $p^{\prime}(0)<0$, hence $p$ has a unique positive root. This root is positive even when $q_{c, i}$ happens to be zero, so that in this situation the formation of droplets is initiated.

(b) In the non-supersaturated regime $c$ is nonpositive and $p$ is strictly monotonically increasing for $x \geq 0$. Accordingly, $p$ has a unique nonnegative root.

(b1) If $q_{c, i}=0$, then $q_{c, i+1}=0$, too; no droplets are generated in this case.

(b2) If $q_{c, i}>0$, then $p(0)<0$, and hence $q_{c, i+1}$ is strictly positive. Moreover, $q_{c, i+1}$ is strictly smaller than $q_{c, i}$ in this case, since

$$
p\left(q_{c, i}^{\frac{1}{3}}\right)=q_{c, i}-q_{c, i}+\tau\left(a_{1} q_{c, i}^{2}+a_{2} q_{c, i}-c q_{c, i}^{\frac{1}{3}}\right)>0,
$$

because the term in parantheses is strictly positive.

Newton's method

$$
x_{k+1}=x_{k}-\frac{p\left(x_{k}\right)}{p^{\prime}\left(x_{k}\right)}, \quad k=0,1,2, \ldots,
$$

is the method of choice for computing the positive root of (37) in the cases (a) and (b2) efficiently.

(a) In the supersaturated regime we recommend to choose

$$
x_{0}=x_{0, \mathrm{a}}=\min \left\{\left(\frac{\tau c}{3}\right)^{\frac{1}{2}},\left(\frac{c}{6 a_{1}}\right)^{\frac{1}{5}}\right\},
$$

because $p^{\prime}\left(x_{0}\right) \geq 0$ in this case, and this guarantees quadratic convergence of the Newton iteration. 


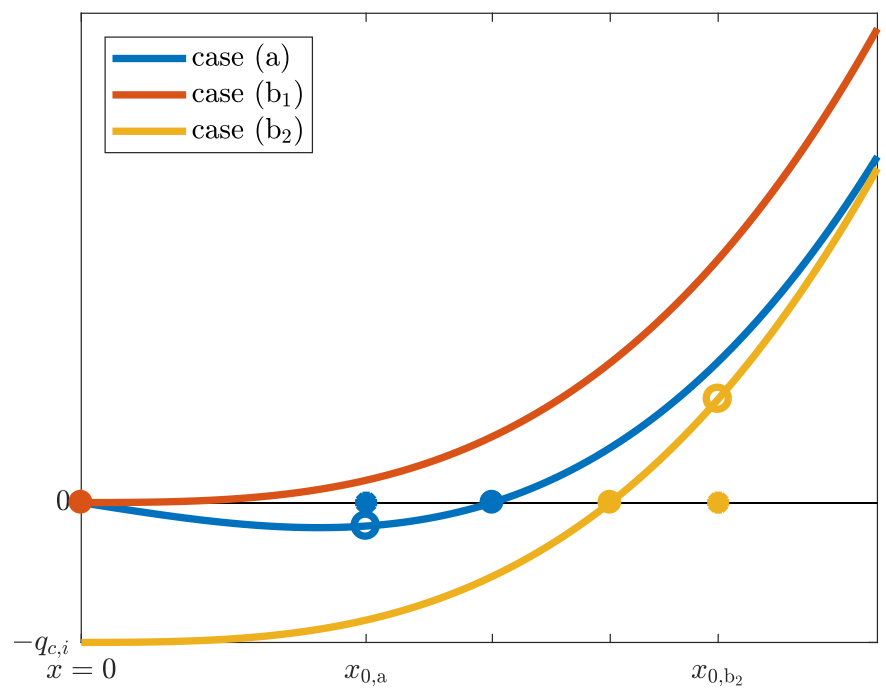

Figure 4: Illustration of the shape of $p$ for Newton's method.

(b2) In the non-supersaturated regime we suggest to take

$$
x_{0}=x_{0, \mathrm{~b} 2}=\left(\frac{q_{c, i}}{\tau a_{2}+1}\right)^{\frac{1}{3}},
$$

because $p\left(x_{0}\right)>0$ for this choice, and again, this guarantees quadratic convergence of the Newton iteration.

The two initial guesses (38) and (39) are indicated by circles in Figure 4.

\subsection{Remaining problems}

Here we address a few peculiarities that may arise in numerical simulations.

\subsubsection{Vanishing rain drop quantities}

Due to round-off it may happen that one of the two variables $q_{r}$ and $n_{r}$ has become zero at some point, while the other one is still positive. In that case we naturally set the associated evaporation term $E$ or $E^{\prime}$, respectively, to zero, because the corresponding quantity cannot evaporate as it is not present. It then follows from the respective implicit Euler equation (27) or (28) that this variable stays zero at the intermediate time step $i+1 / 2$.

If $n_{r, i}=0$, then we also conclude from (15) that $E=0$, and hence we obtain

$$
q_{r, i+1 / 2}=\frac{1}{1+\tau s} q_{r, i}, \quad n_{r, i+1 / 2}=0 ; \quad E=0 ; \quad S_{\text {out }}=s q_{r, i+1 / 2}, \quad S_{\text {out }}^{\prime}=0 ;
$$

this is correct, independent of whether $q_{r, i}=0$ as well, or not.

On the other hand, if $q_{r, i}=0$, but $n_{r, i} \neq 0$, then formally, $1 / \bar{m}_{r}=+\infty$ in (33), so that the evaporation rate $E^{\prime}$ is maximal; cf. (14). We take this as reasoning to completely "evaporate" the remaining number concentration in one single time step, and to set $n_{r, i+1}$ and the associated sedimentation term to zero, i.e.,

$$
q_{r, i+1 / 2}=0, \quad n_{r, i+1 / 2}=0 ; \quad E=0 ; \quad S_{\text {out }}=S_{\text {out }}^{\prime}=0 .
$$

Note that the value of $E^{\prime}$ is irrelevant for the remaining computations in this time step. 


\subsubsection{Negative vapour concentrations}

In the supersaturated regime it may happen that the condensation term becomes larger than the available vapour mass when the time step is too large. Although this is not a very realistic scenario because in this regime $q_{v}$ will rarely be sufficently small for this to happen, this may lead to a negative value of $q_{v, i+1}$.

One option to cure this problem is to set $q_{v, i+1}=0$ in this case, but this would result in a gain of water mass. We therefore recommend to reject such a time step instead, and to repeat the computation with a smaller time step.

\subsubsection{Updating the box size}

As mentioned before, cf. (32), at the very end of each time step we need to modify the height of every box to compensate for changes in the density. In the column model this leads to additional vertical movements $\Delta w$ of all but the lowermost boxes, and this in turn gives rise to a corresponding change

$$
\Delta p=-\gamma \rho \Delta w, \quad \Delta T=-\gamma \Delta w,
$$

of pressure and temperature; cf. (24). Different to what has been said right before Section 3.1 we therefore need to update the pressure and the temperature according to (40) right at the beginning of the subsequent time step, even before the other parameters - some of which depend on $p$ or $T$ - are being evaluated.

\section{Numerical simulations}

In this section we present some numerical experiments with the new model. In Section 4.1 we demonstrate that our new model reproduces reasonable levels of supersaturation, while Section 4.2 shows the negative impact of using saturation adjustment instead. In Section 4.3 we compare our cloud activation model with a more sophisticated scheme, which is based on an effective CCN distribution. While these first three experiments use the box model, the last experiment described in Section 4.4 considers the full column model and demonstrates that it determines reasonable amounts of precipitation for three representative updraft scenarios.

\subsection{Time evolution of supersaturation}

As a first test case we have compared our time evolution of supersaturation with the results published by [20]. To this end we have reduced our model to the process $C$ of condensation, i.e., collision and sedimentation processes have been switched off. In addition, we have prescribed a fixed number concentration of cloud droplets. This setup has been used for a direct comparison with [20] for different sources of supersaturation, i.e., different vertical upward motions, which drive the adiabatic cooling and thus provide a permanent source for supersaturation.

The simulation starts in a regime with $q_{v s}-q_{v}=0, T(0)=273.15 \mathrm{~K}$ and $p(0)=87000 \mathrm{~Pa}$ with $\rho n_{c}=2 \cdot 10^{8} \mathrm{~m}^{-3}$ cloud droplets that have the initial radius of $5 \mu \mathrm{m}$. These droplets grow by condensation while their amount $n_{c}$ is kept constant. Different vertical velocities are used for representing different degrees of supersaturation, i.e., $w \in\{0.25,0.5,1,2\} \mathrm{m} \mathrm{s}^{-1}$. Figure 5 shows the results, which agree almost perfectly with the supersaturation behavior shown by [20, their Figure 1]. Especially, the time evolution of the supersaturation with a peak and a subsequent decay can be seen. Although supersaturation is known to be quite sensitive to changes in the parameters and therefore also to numerical errors, our semi-implicit strategy is well suited for the approximation of supersaturation. 


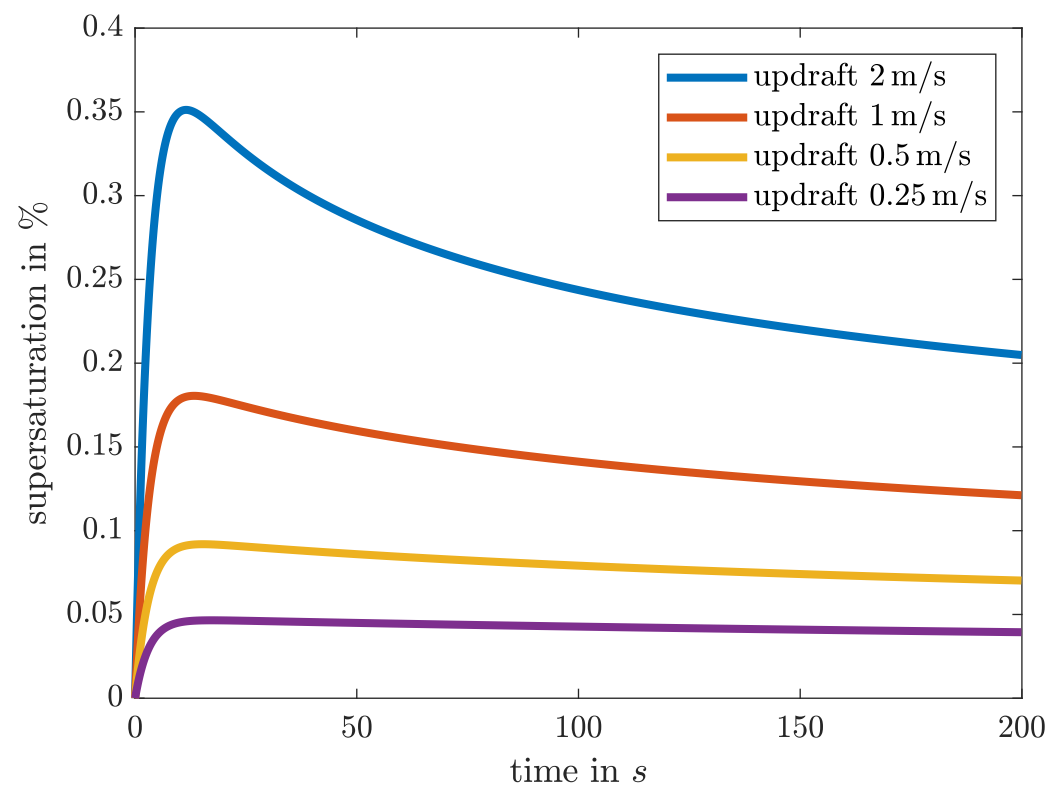

Figure 5: Temporal evolution of the supersaturation ratio $q_{v} / q_{v s}$ as computed with our cloud model for different updraft velocities. The cloud model reproduces the results of [20] almost perfectly.

\subsection{The impact of saturation adjustment}

One of the major disadvantages of saturation adjustment shows up in its influence on cloud buoyancy. The potential density temperature

$$
\theta_{d}:=\theta\left(1+\varepsilon_{0} q_{v}-q_{c}\right), \quad \theta=T\left(\frac{p_{0}}{p}\right)^{\frac{R_{a}}{C_{p}}}, \varepsilon_{0}=\frac{1}{\varepsilon}-1 \approx 0.608
$$

in the air parcel and its difference to the corresponding quantity of the environment determines the buoyancy and thus the time evolution of vertical velocities. If saturation adjustment is introduced, more latent heat will be produced, which results in higher updraft velocities and stronger cooling of the air parcel, and hence to further condensation, a vicious cycle. In this section we want to quantify the impact on $\theta_{d}$ if our model would use saturation adjustment instead of tolerating supersaturation. This study is similar in nature to one by [8], although the feedback effect cannot be treated in our simple box model, because our upward motion is predefined.

For the implementation of saturation adjustment we have formulated a complementarity problem for $q_{c}+q_{v}$, which is then solved numerically using a Newton scheme. This replaces the nonlinear equation (29), and thus corresponds to a different activation scheme.

We have set up an experiment in a maritime environment, i.e., using $N_{\infty}=8 \cdot 10^{6} \mathrm{~kg}^{-1}$, an initial temperature of $303.15 \mathrm{~K}$, and an initial pressure of $85000 \mathrm{~Pa}$. We further have prescribed a constant updraft $\left(w=10 \mathrm{~m} \mathrm{~s}^{-1}\right.$ ) with an air parcel at water saturation, but without any liquid water. In our model the parcel is then permanently supersaturated, while the alternative model with saturation adjustment keeps it at saturation at any time. The time evolution of water vapour concentration $q_{v}$ and the potential density temperature $\theta_{d}$ of the two methods are shown in Figure 6. It is clear that with saturation adjustment more water vapour is depleted and more latent heat is released as compared to our model. Therefore, the increase of potential density temperature is more pronounced for saturation adjustment: After $t \sim 10 \mathrm{~s}$ the resulting difference is $\Delta \theta_{d} \approx 0.3 \mathrm{~K}$. Although this seems quite marginal, it will already introduce a non-negligible additional buoyancy as has been demonstrated in [8]. This supports our modeling decision to develop a cloud scheme without saturation adjustment. 

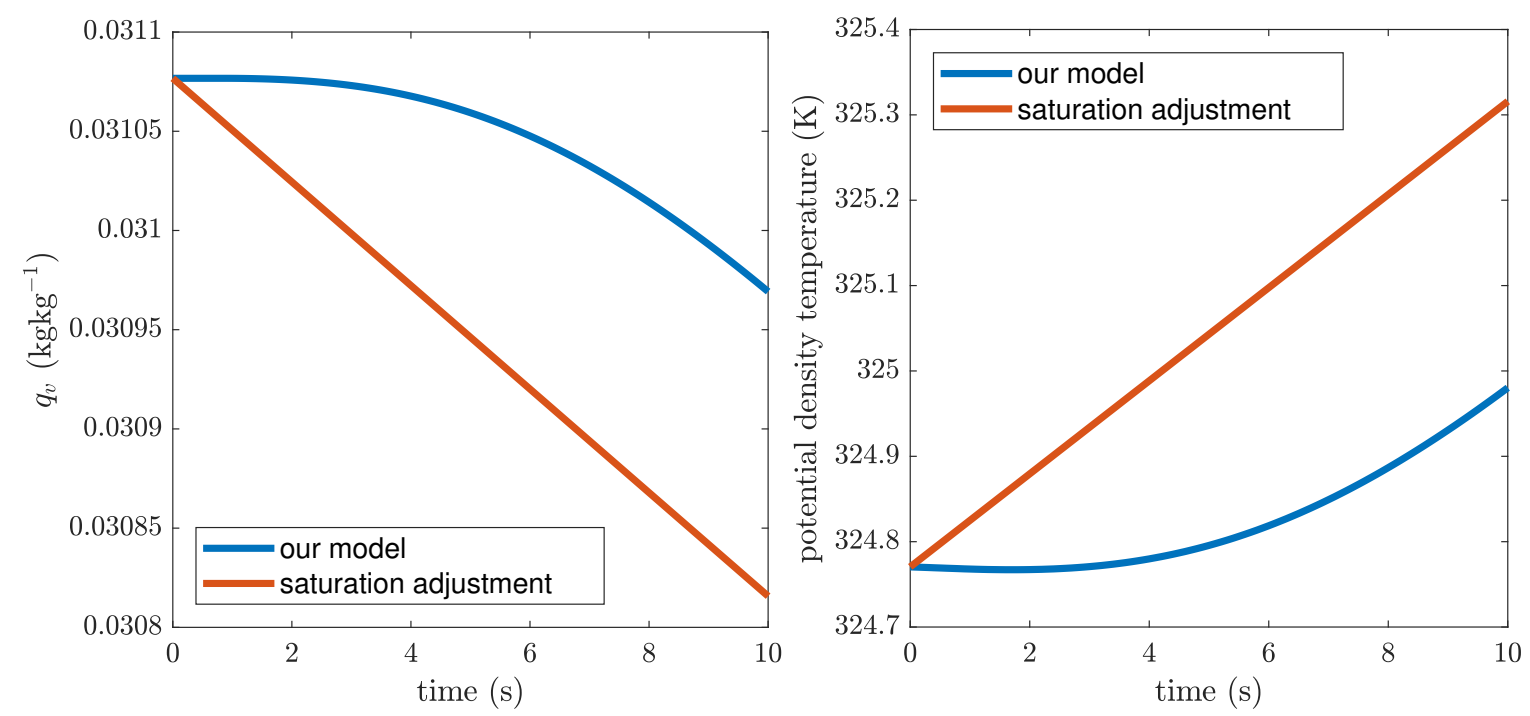

Figure 6: Evolution of water vapour concentration $q_{v}$ (left) and potential density temperature $\theta_{d}$ (right) for the two schemes.

\subsection{Activation of cloud droplets}

Next we show that our strategy of taking the cloud droplet number concentration to be a nonlinear function of the droplet mass concentration yields reasonable droplet activation counts, by comparing our model with a sophisticated activation scheme. To this end, we have switched off the processes $A_{1}$ and $A_{2}$, and have focused on the activation of cloud droplets within a continental regime (high amount of CCNs) and a maritime environment (low amount of CCNs).

\subsubsection{Activation schemes}

The sophisticated reference model uses a two moment scheme for a single air parcel, i.e., it explicitely tracks the number and mass concentrations $n_{c}$ and $q_{c}$ of the cloud droplets together with the evolution of pressure, temperature, and saturation ratio $\frac{q_{v}}{q_{v s}}$. The equations for pressure, temperature, and the condensation process are the same as in (24) and (23), respectively. In addition to the condensation process, the differential equation for $q_{c}$ includes the change in number concentration by assuming a constant mass for a newly activated droplet with radius $0.5 \mu \mathrm{m}$, resembling the choice of $m_{0}$ in (22), see also Table 4.

The equation for the number concentration $n_{c}$ in the two-moment scheme is given by

$$
\dot{n}_{c}=\frac{1}{\tau_{\mathrm{act}}}\left(N_{\mathrm{CCN}}-n_{c}\right)_{+} \frac{\left(q_{v}-q_{v s}\right)_{+}}{q_{v}-q_{v s}}
$$

with an activation timescale $\tau_{\text {act }}=1 \mathrm{~s}$ and a CCN spectrum $N_{\mathrm{CCN}}$ which depends on the saturation ratio $q_{v} / q_{v s}$, following [27]. The last factor in (42) ensures that the number concentration can only change in the supersaturated regime. For the choice of the CCN spectrum $N_{\mathrm{CCN}}$ there are essentially two possibilities [see 12]. The first possibility is based on a background aerosol particle distribution, resulting in a detailed, but expensive scheme based on Köhler theory. The second possibility, as is employed here, is the choice of an empirical relationship, in particular a power-law relation ("Twomey spectrum")

$$
N_{\mathrm{CCN}}=C_{\mathrm{CCN}}\left(q_{v} / q_{v s}-1\right)^{\kappa}
$$

with positive parameters $C_{\mathrm{CCN}}$ and $\kappa$. The choice of $C_{\mathrm{CCN}}$ encodes the typical background aerosol number (being different for continental and maritime scenarios) and the exponent $\kappa<1$ adjusts the steepness of $N_{\mathrm{CCN}}$ near $q_{v}=q_{v s}$, i.e., the sensitivity of $N_{\mathrm{CCN}}$ for small levels of supersaturation. 
Table 1: Relative root mean square errors for the maritime case, depending on the choice of $N_{\infty}$.

\begin{tabular}{rcc}
\hline$N_{\infty}\left[\mathrm{kg}^{-1}\right]$ & saturation ratio & $q_{c}$ \\
\hline $6 \cdot 10^{7}$ & $5.61 \cdot 10^{-4}$ & 0.0167 \\
$8 \cdot 10^{7}$ & $3.88 \cdot 10^{-4}$ & 0.0199 \\
$10 \cdot 10^{7}$ & $3.58 \cdot 10^{-4}$ & 0.0240 \\
\hline
\end{tabular}

Table 2: Relative root mean square errors for the continental case, depending on the choice of $N_{\infty}$.

\begin{tabular}{rcc}
\hline$N_{\infty}\left[\mathrm{kg}^{-1}\right]$ & saturation ratio & $q_{c}$ \\
\hline $6 \cdot 10^{8}$ & $2.09 \cdot 10^{-3}$ & 0.0189 \\
$8 \cdot 10^{8}$ & $8.00 \cdot 10^{-4}$ & 0.0198 \\
$10 \cdot 10^{8}$ & $1.20 \cdot 10^{-4}$ & 0.0206 \\
\hline
\end{tabular}

\subsubsection{Maritime and continental scenarios}

In the following, we consider a continental and a maritime case to compare our cloud model with the detailed two moment scheme described in the preceding section. In both cases we consider a single air parcel, ascending with $2 \mathrm{~m} \mathrm{~s}^{-1}$ with initial pressure $87000 \mathrm{~Pa}$ and temperature $273.15 \mathrm{~K}$. Since we want to compare the activation of cloud droplets, we assume an initial humidity $q_{v}=q_{v s}$, i.e. the air parcel is initially at saturation. The time step in both models is $\tau=0.01 \mathrm{~s}$. As explained in Section 2.4.1, in our new cloud model the number of cloud droplets is tied to the mass concentration $q_{c}$ through (22). Therefore, we choose the initial number concentration for the two moment scheme according to this relation and assume in both models $q_{c}(0)=10^{-10} \mathrm{~kg} \mathrm{~kg}^{-1}$, i.e. an essentially cloud free case. The choices for $N_{0}$ and $m_{0}$ are given in Table 4.

For the maritime case, the parameter choices for the CCN-spectrum (43) are given by $C_{\mathrm{CCN}}=9 \cdot 10^{8} \mathrm{~kg}^{-1}$ and $\kappa=\frac{1}{2}$, so that at $1 \%$ supersaturation the number concentration of cloud droplets corresponds to the figure of $100 \mathrm{~cm}^{-3}$ tabulated in [34]. The free parameter $N_{\infty}$ in (22) is selected from the interval $6 \cdot 10^{7} \mathrm{~kg}^{-1} \leq$ $N_{\infty} \leq 1 \cdot 10^{8} \mathrm{~kg}^{-1}$. Figure 7 shows the saturation ratio $q_{v} / q_{v s}$ (right panel), the mass concentration (middle panel), and the number concentration (left panel) for the two moment scheme (blue curves) and the new cloud model (red curves) with parameter $N_{\infty}=8 \cdot 10^{7} \mathrm{~kg}^{-1}$. We observe a good agreement of the supersaturation ratio and the cloud droplet mass concentration for both schemes, see also Table 1 .

For the continental case, we choose the parameters $C_{\mathrm{CCN}}=4.69 \cdot 10^{9} \mathrm{~kg}^{-1}$ and $\kappa=0.308$ in (43), so that at $1 \%$ supersaturation the number concentration of cloud droplets is corresponding to $1260 \mathrm{~cm}^{-3}$ [as given in 37]. Here we vary $N_{\infty}$ between $6 \cdot 10^{8} \mathrm{~kg}^{-1}$ and $1 \cdot 10^{9} \mathrm{~kg}^{-1}$ because the number of CCN is almost always one order of magnitude larger over land than over sea [34]. Figure 8 shows the corresponding results with the choice $N_{\infty}=8 \cdot 10^{8} \mathrm{~kg}^{-1}$ : saturation ratio (right panel), mass concentration (middle panel), and number concentration (left panel). Although our cloud model slightly overestimates the saturation ratio, we again observe a good agreement for the mass and number concentrations, see Table 2.

It is impressive that the fixed coupling of mass and number of the cloud droplets yields such a good agreement of our numerical results with those of the much more detailed two moment scheme, where the number and mass concentrations evolve independently. In our model, we only have adjusted the parameter $N_{\infty}$ in (22) to the given regime (maritime or continental). Keeping this in mind, the agreement of the saturation ratio between our cloud model and the two moment scheme is surprisingly good, given the sensitivity of the saturation ratio with respect to changes in the modeling of the condensation process and the mass concentration. 

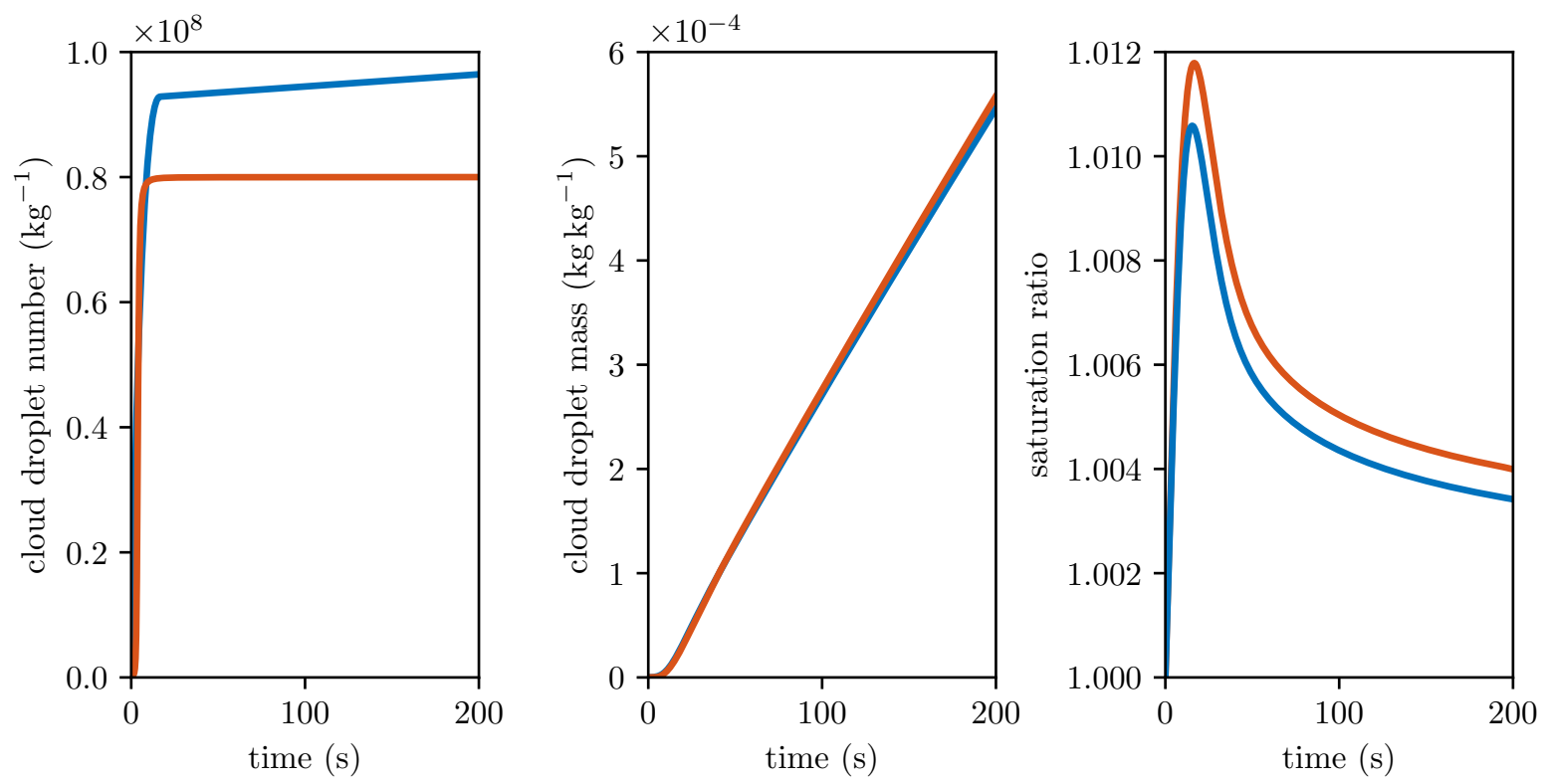

Figure 7: Maritime test case: our scheme (red) versus reference scheme (blue)

\subsection{The full column model: Three updraft scenarios}

Finally we have run the full column model for the following three updraft scenarios: a warm front with small vertical velocity $0.05 \mathrm{~m} \mathrm{~s}^{-1}$, a warm conveyor belt with moderate vertical velocity $0.5 \mathrm{~m} \mathrm{~s}^{-1}$, and a convective event with large vertical velocity $5 \mathrm{~m} \mathrm{~s}^{-1}$. In each case we have assumed that the column does not ascend in the first $5 \mathrm{~min}$; thereafter the vertical velocity has been set to the respective value of this scenario until the bottom of the column has reached a height of $1500 \mathrm{~m}$, where the vertical velocity has been set to zero again, see Figure 9. The column consists of five air parcels, each with an initial height of $200 \mathrm{~m}$. For each scenario, the initial conditions as well as the saturation profiles are identical: The initial temperature and pressure for the lowermost air parcel are $300 \mathrm{~K}$ and $101325 \mathrm{~Pa}$, respectively; for the upper air parcels the temperature and pressure have been initialized according to the adiabatic lapse rates. The lower three air parcels are initially subsaturated with $40 \%$ water saturation, while the upper two air parcels have initially $80 \%$ saturation. With this setup, the temperature in the whole column never falls below $275 \mathrm{~K}$ during our simulation, hence there occurs no ice, so that our cloud scheme is a reasonable model.

Figure 10 shows the corresponding precipitation rates as a function of time. The different amounts of rain for the three cases is realistic: The convective event produces the largest rainfall in a short time, being in-line with the large updraft velocity. In contrast, the case of a warm front with very small updraft velocity produces after about $4 \mathrm{~h}$ only light but steady rainfall. Rainfall for the warm conveyor belt is more similar to the convective case, but with an initial delay of roughly half an hour, and also the amplitude is smaller. The relatively small total precipitation rates in all cases are due to the subsaturated lower three air parcels of the column. These air parcels remain cloud free during the whole simulation and do not produce any rain at all, while the upper two air parcels are cloudy and produce all of the rain seen in Figure 10.

As already mentioned, the lower part of the column is relatively dry and gets moistened by the evaporation of rain, falling down from the cloudy upper part. This effect is illustrated in Figure 11 for the case of the warm conveyor belt. The blue line shows the temporal evolution of the saturation ratio at the bottom of the upper cloudy part of the column. Due to the updraft, this part gets supersaturated after roughly $20 \mathrm{~min}$ and the cloud forms. The rainfall starts after $40 \mathrm{~min}$ and reaches its maximum at roughly $60 \mathrm{~min}$, see the red curve in Figure 10. This indicates the delay in the production of the rain due to the autoconversion process. After falling out of the cloudy part of the column, the rain falls through the subsaturated lower part of the column and evaporates. To illustrate the moistening of the lower part we have added the yellow and the red curve in Figure 11: While the yellow curve displays the actual saturation ratio in the lower part of the column, 

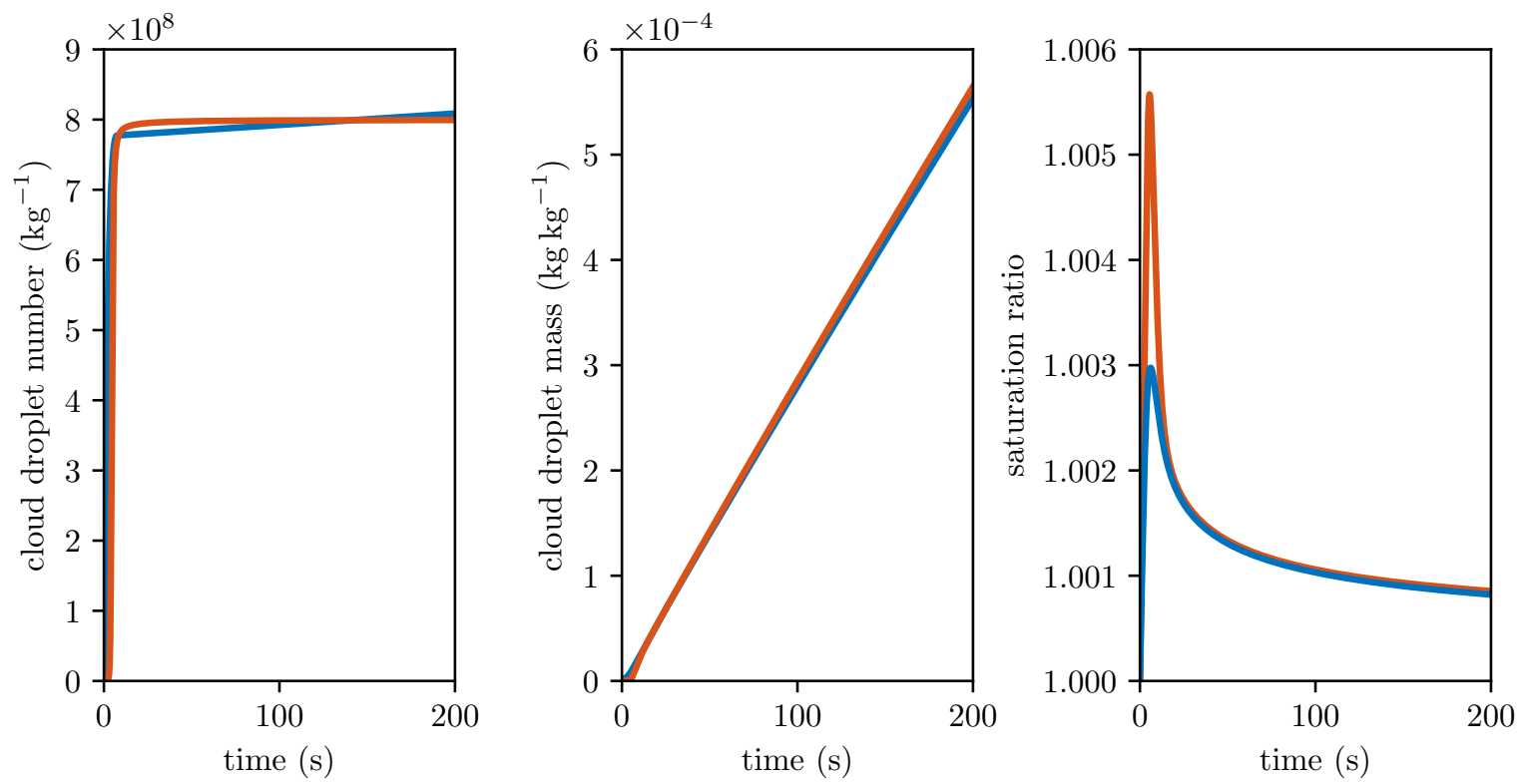

Figure 8: Continental test case: our scheme (red) versus reference scheme (blue)

the red curve shows the corresponding data, if the evaporation of rain were switched off. Consequently, the difference of these curves indicates the moistening of the lower part of the column.

In Sections 2.7 and 3.2 we have discussed the conservation of the total water mass in our cloud model, when one accounts for the amount of rain falling out of the column. The absolute loss of total water mass that we have observed in our simulations are $2.19 \cdot 10^{-14} \mathrm{~kg} \mathrm{~kg}^{-1}$ for the warm front, $3.97 \cdot 10^{-15} \mathrm{~kg} \mathrm{~kg}^{-1}$ for the warm conveyor belt, and $1.57 \cdot 10^{-15} \mathrm{~kg} \mathrm{~kg}^{-1}$ for the convective case. These values confirm the conservation of mass numerically.

\section{Summary and conclusions}

In this paper we have developed a new model for warm clouds based on averaged mass and number concentrations of cloud droplets and rain drops. The model consists of a one-and-a-half moment scheme with differential equations for the mass concentration of cloud droplets, and the mass and number concentrations for rain drops. To account for a realisitic activation of cloud droplets we do not use a differential equation for the droplet number concentration, but we couple the droplet number and mass concentrations directly with a nonlinear functional relation instead. Growth and evaporation of cloud droplets are realized in such a way that a certain level of supersaturation with respect to water is tolerated, i.e., we do not apply any sort of saturation adjustment. The collision terms are given by rates, which are nonlinear in the mass concentrations, similar to previous model developments. The sedimentation of rain drops is formulated for a zero-dimensional box model and for a vertical column model.

For the implementation of this model we propose a consistent numerical scheme, which is semi-implicit, i.e., some terms are treated explicitly while others are solved for implicitly. Implicit solvers are necessary, for example, to activate cloud droplets, because for zero initial conditions explicit solvers will always stick to the nonphysical trivial solution. The corresponding implicit equation is solved using Newton's method, assuring a quadratic convergence. The implementation is proven to return nonnegative concentrations only, and to preserve the air and water mass balance.

The model has been successfully tested on idealized model setups. We have compared our numerical results with reference data for the time evolution of supersaturation presented in [20]. In addition, we have demonstrated the negative effect of saturation adjustment as compared to our model formulation. We have 


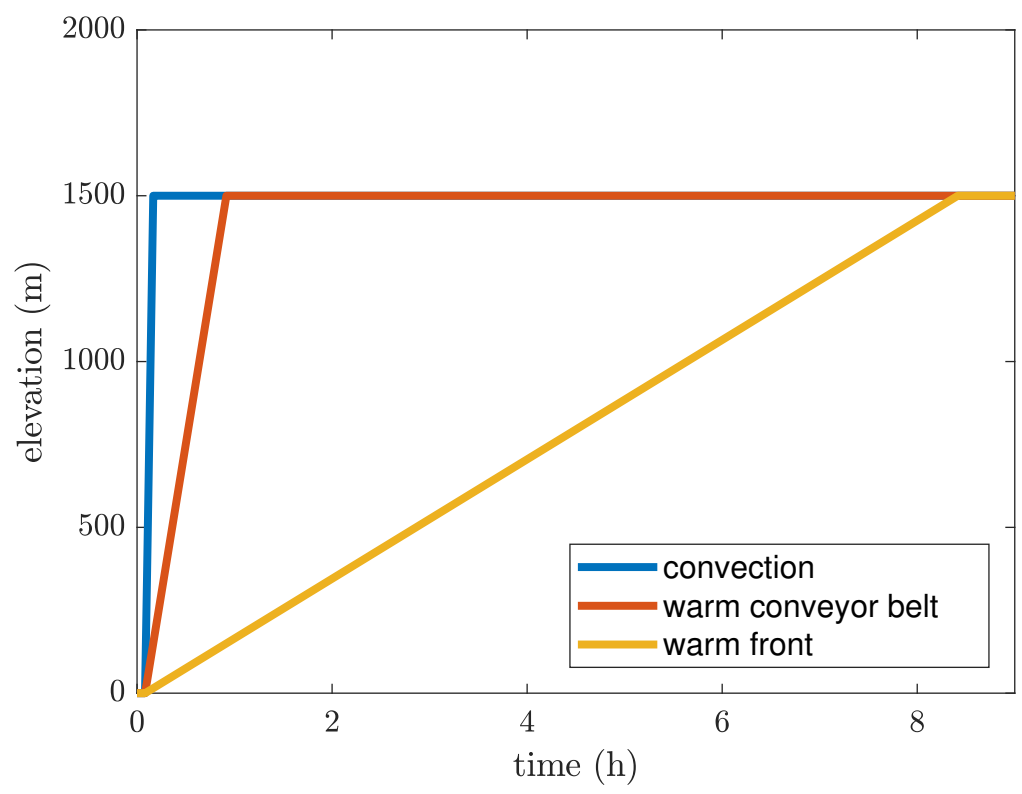

Figure 9: Ascend of the lowermost air parcel of the column over time for the three scenarios.

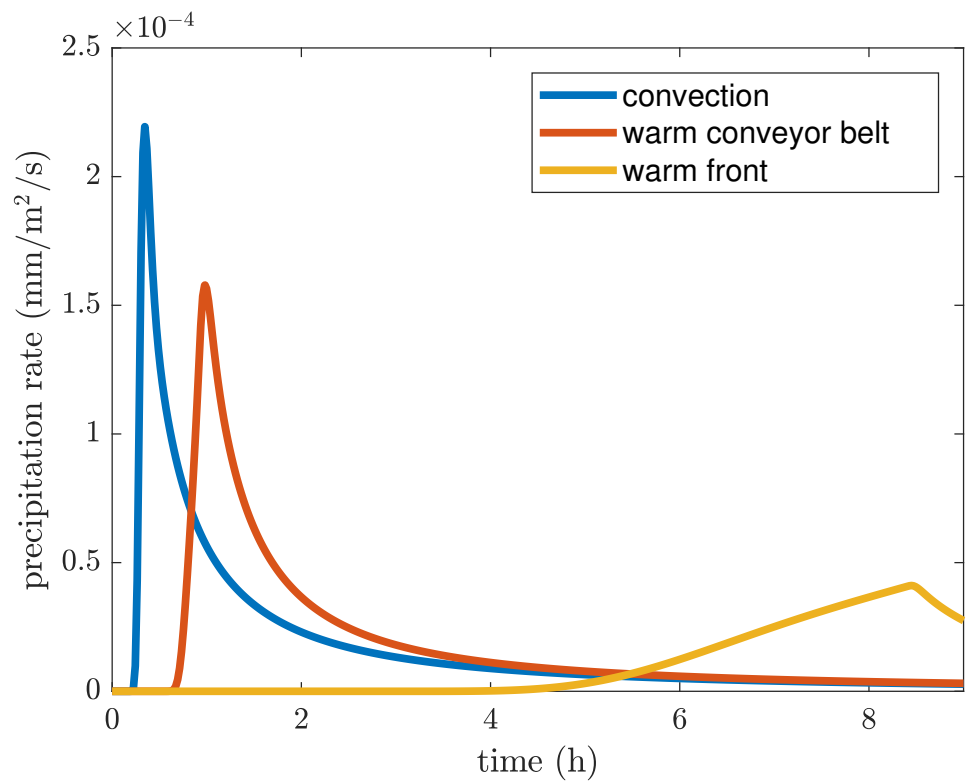

Figure 10: Precipitation rates as a function of time. 


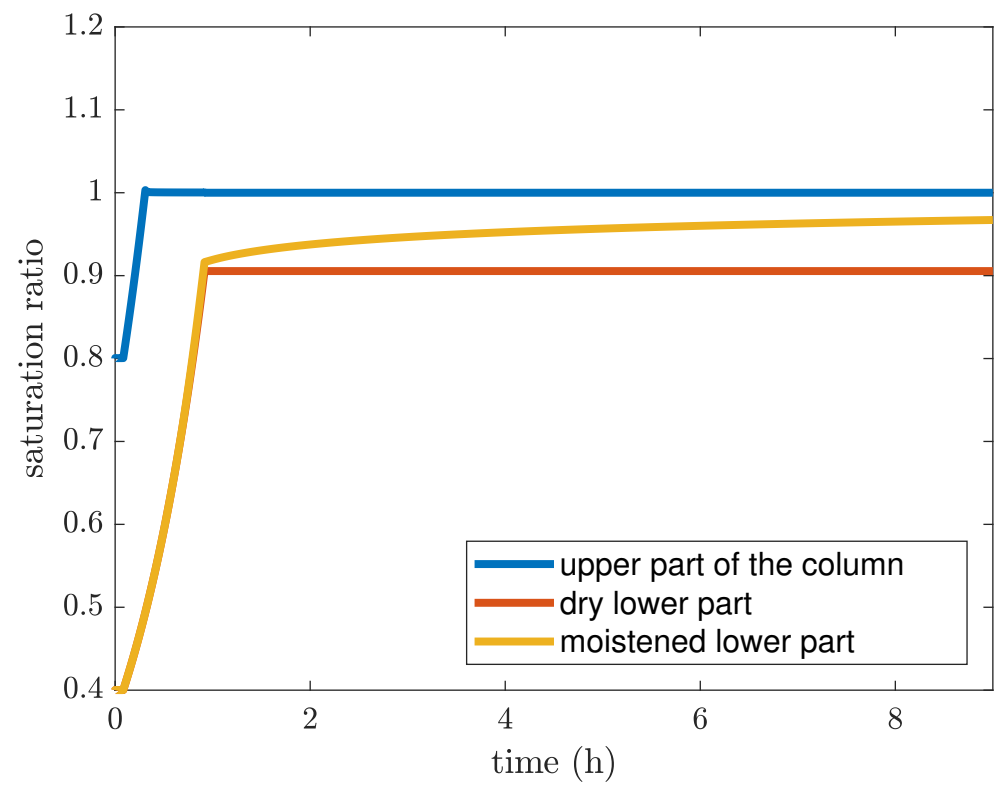

Figure 11: Saturation ratio of the air parcels within the column for the warm conveyor belt. Blue curve: upper two air parcels of the column; yellow curve: lower part of the column; red curve: lower part of the column without the moisture of the evaporating rain.

also implemented and run a more sophisticated explicit droplet activation scheme for two different meteorological scenarios (continental vs. maritime environment): In both cases we have seen a very good agreement of our scheme. Finally, we have also shown that our column model determines meaningful rain formation pathways and precipitation data for different updraft scenarios, such as slow frontal updraft, warm conveyor belts, and convective events, respectively.

Acknowledgement: We thank the two anonymous reviewers for their helpful comments on the manuscript. Nikolas Porz acknowledges support of the Transregional Collaborative Research Center SFB/TRR 165 "Waves to Weather", funded by the Deutsche Forschungsgemeinschaft (DFG), within the subproject "Identification of robust cloud patterns via inverse methods" (B7). Manuel Baumgartner acknowledges support of the Deutsche Forschungsgemeinschaft (DFG) within the project "Enabling Performance Engineering in Hesse and Rhineland-Palatinate" (grant number 320898076). We thank Philipp Reutter for interesting discussions of our droplet activation process. We thank Klaus Gierens for remembering and providing a reference for an inequality of moments.

\section{A Technical details of the overall algorithm}

A flowchart of the numerical algorithm is presented in Figure 12. The physical constants and the model parameters are summarized in Tables 3 and 4, respectively. Finally, we use the parameterization

$$
\begin{aligned}
\log \left(p_{\mathrm{s}}(T)\right)= & 54.842763-6763.22 / T-4.210 \log (T)+0.000367 T \\
+ & \tanh (0.0415(T-218.8)) \\
& \cdot(53.878-1331.22 / T-9.44523 \log (T)+0.014025 T)
\end{aligned}
$$

for the saturation vapour pressure over a flat surface of water from [31]. 


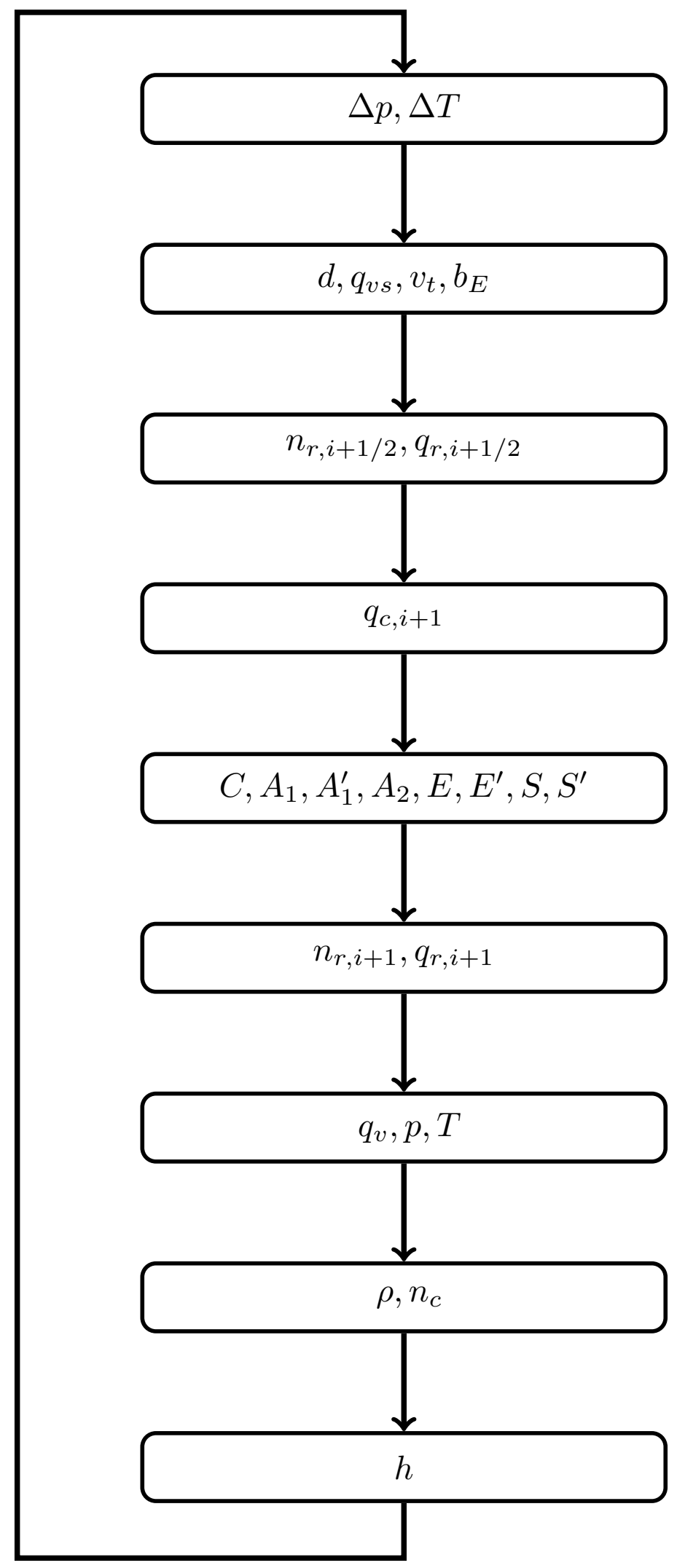

Figure 12: Flowchart of the numerical algorithm for the integration of the cloud model. 
Table 3: Physical constants and reference quantities.

\begin{tabular}{ll}
\hline Constant & Description \\
\hline$p_{\star}=101325 \mathrm{~Pa}$ & reference pressure \\
$T_{\star}=288 \mathrm{~K}$ & reference temperature \\
$T_{0}=273.15 \mathrm{~K}$ & melting temperature \\
$\rho_{\star}=1.225 \mathrm{~kg} \mathrm{~m}^{-3}$ & reference air density \\
$\gamma=\frac{g}{c_{p}}=0.00976 \mathrm{~K} \mathrm{~m}^{-1}$ & dry adiabatic lapse rate \\
$\rho_{l}=1000 \mathrm{~kg} \mathrm{~m}^{-3}$ & density of liquid water \\
$R_{a}=287.05 \mathrm{~J} \mathrm{~kg}^{-1} \mathrm{~K}^{-1}$ & specific gas constant, dry air \\
$R_{V}=461.52 \mathrm{~J} \mathrm{~kg}^{-1} \mathrm{~K}^{-1}$ & specific gas constant, water vapour \\
$C_{p}=1005 \mathrm{~J} \mathrm{~g}^{-1} \mathrm{~K}^{-1}$ & specific heat capacity, dry air \\
$g=9.81 \mathrm{~m} \mathrm{~s}^{-2}$ & acceleration due to gravity \\
$L=2.53 \cdot 10^{6} \mathrm{~J} \mathrm{~kg}^{-1}$ & latent heat of vapourisation \\
$\mathcal{E}=\frac{M_{\mathrm{mol}, v}}{M_{\mathrm{mol}}, a}=0.622$ & ratio of molar masses of water and dry air \\
$D_{0}=2.11 \cdot 10^{-5} \mathrm{~m}^{2} \mathrm{~s}^{-1}$ & diffusivity constant \\
\hline
\end{tabular}

Table 4: Model parameters.

\begin{tabular}{ll}
\hline Parameter & Description \\
\hline$\alpha=190.3 \mathrm{~m} \mathrm{~s}^{-1} \mathrm{~kg}^{-\beta}$ & parameter for terminal velocity \\
$\beta=\frac{4}{15}$ & parameter for terminal velocity \\
$m_{t}=1.21 \cdot 10^{-5} \mathrm{~kg}$ & parameter for terminal velocity \\
$k_{1}=0.0041 \mathrm{~kg} \mathrm{~s}^{-1}$ & parameter for autoconversion \\
$k_{2}=0.8 \mathrm{~kg}$ & parameter for accretion \\
$N_{0}=1000 \mathrm{~m}^{-3}$ & parameter for activation \\
$N_{\infty}$ & parameter for activation \\
$m_{0}=\frac{4}{3} \pi \rho_{l}(0.5 \mu \mathrm{m})^{3}$ & parameter for activation \\
$a_{E}=0.78$ & parameter for evaporation \\
$a_{v}=0.78$ & parameter for ventilation \\
$b_{v}=0.308$ & parameter for ventilation \\
$c_{q}=1.84$ & parameter for effective fall velocity \\
$c_{n}=0.58$ & parameter for effective fall velocity \\
$h$ & height of the box
\end{tabular}




\section{References}

[1] Sylwester Arabas and Shin-ichiro Shima. On the ccn (de) activation nonlinearities. Nonlinear processes in geophysics, 24(3): 535-542, 2017. 10.5194/npg-24-535-2017.

[2] K. D. Beheng. The Evolution of Raindrop Spectra: A Review of Microphysical Essentials. In F. Y. Testik and M. Gebremichael, editors, Rainfall: State of the Science, Geophysical Monograph Series, pages 29-48, 2010.

[3] B. J. Devenish, P. Bartello, J.-L. Brenguier, L. R. Collins, Wojciech W. Grabowski, R. H. A. IJzermans, Szymon P. Malinowski, M. W. Reeks, J. C. Vassilicos, L.-P. Wang, and Z. Warhaft. Droplet growth in warm turbulent clouds. Quarterly Journal of the Royal Meteorological Society, 138(667):1401-1429, 2012. ISSN 1477-870X. 10.1002/qj.1897. URL http://dx.doi.org/10.1002/ qj.1897.

[4] J. Dixon. The Shock Absorber Handbook. Wiley, 2007. ISBN 978-0-470-51020-9.

[5] G. Doms, J. Förstner, E. Heise, H.-J. Herzog, D. Mironow, M. Raschendorfer, T. Reinhardt, B. Ritter, R. Schrodin, J.-P. Schulz, and G. Vogel. A description of the nonhydrostatic regional cosmo model. part ii: Physical parameterization, 2011.

[6] K. Gierens and S. Bretl. Analytical treatment of ice sublimation and test of sublimation parameterisations in two-moment ice microphysics models. Atmospheric Chemistry and Physics, 9(19):7481-7490, 2009. 10.5194/acp-9-7481-2009. URL https://www.atmos-chem-phys.net/9/7481/2009/.

[7] Wojciech W. Grabowski. Untangling microphysical impacts on deep convection applying a novel modeling methodology. Journal of the Atmospheric Sciences, 72(6):2446-2464, 2015. 10.1175/JAS-D-14-0307.1.

[8] Wojciech W. Grabowski and Dorota Jarecka. Modeling condensation in shallow nonprecipitating convection. Journal of the Atmospheric Sciences, 72(12):4661-4679, 2015. 10.1175/JAS-D-15-0091.1.

[9] Wojciech W. Grabowski and Hugh Morrison. Untangling microphysical impacts on deep convection applying a novel modeling methodology. part ii: Double-moment microphysics. Journal of the Atmospheric Sciences, 73(9):3749-3770, 2016 10.1175/JAS-D-15-0367.1.

[10] Wojciech W. Grabowski and Hugh Morrison. Modeling condensation in deep convection. Journal of the Atmospheric Sciences, 74(7):2247-2267, 2017. 10.1175/JAS-D-16-0255.1.

[11] WW Grabowski. A parameterization of cloud microphysics for long-term cloud-resolving modeling of tropical convection. Atmospheric Research, 52(1-2):17-41, 1999. 10.1016/S0169-8095(99)00029-0.

[12] Fritz Herbert and Ulrike Wacker. Parameterization of the ccn-humidity spectrum in dependency on nucleation conditions and aerosol size distribution. Meteorology and Atmospheric Physics, 68(3):213-220, 1998. ISSN 1436-5065. 10.1007/BF01030212. URL http://dx.doi.org/10.1007/BF01030212.

[13] Adele L. Igel and Susan C. van den Heever. The role of latent heating in warm frontogenesis. Quarterly Journal of the Royal Meteorological Society, 140(678, A):139-150, JAN 2014. 10.1002/qj.2118.

[14] $\mathrm{H}$. Joos and $\mathrm{H}$. Wernli. Influence of microphysical processes on the potential vorticity development in a warm conveyor belt: a case-study with the limited-area model cosmo. Quarterly Journal of the Royal Meteorological Society, 138(663):407-418, 2012. 10.1002/qj.934. URL https://rmets.onlinelibrary.wiley.com/doi/abs/10.1002/qj.934.

[15] Edwin Kessler. On the distribution and continuity of water substance in atmospheric circulations., volume 32 of Meteorological Monographs. American Meteorological Society, Boston, 1969.

[16] Alexander P. Khain, M. Ovtchinnikov, M. Pinsky, A. Pokrovsky, and H. Krugliak. Notes on the state-of-the-art numerical modeling of cloud microphysics. Atmospheric Research, 55(3-4):159 - 224, 2000. ISSN 0169-8095. http://dx.doi.org/10.1016/S01698095(00)00064-8. URL http://www.sciencedirect.com/science/article/pii/S0169809500000648.

[17] VI Khvorostyanov. Mesoscale processes of cloud formation, cloud-radiation interaction, and their modeling with explicit cloud microphysics. Atmospheric Research, 39(1-3):1-67, 1995. 10.1016/0169-8095(95)00012-G.

[18] YL Kogan and WJ Martin. Parameterization of bulk condensation in numerical cloud models. Journal of the Atmospheric Sciences, 51(12):1728-1739, 1994. 10.1175/1520-0469(1994)051<1728:POBCIN>2.0.CO;2.

[19] H Köhler. The nucleus in and the growth of hygroscopic droplets. Transactions of the Faraday Society, 32(2):1152-1161, 1936. $10.1039 / \mathrm{tf} 9363201152$.

[20] AV Korolev and IP Mazin. Supersaturation of water vapor in clouds. Journal of the Atmospheric Sciences, 60(24):2957-2974, 2003. 10.1175/1520-0469(2003)060<2957:SOWVIC >2.0.CO;2.

[21] Y.L. Lin, R.D. Farley, and H.D. Orville. Bulk parameterization of the snow field in a cloud model. Journal of Climate and Applied Meteorology, 22(6):1065-1092, 1983. 10.1175/1520-0450(1983)022<1065:BPOTSF 2.0.CO;2.

[22] J.Y. Liu and H.D. Orville. Numerical modeling of precipitation and cloud shadow effects on mountain-induced cumuli. Journal of the Atmospheric Sciences, 26(6):1283-1298, 1969. 10.1175/1520-0469(1969)026<1283:NMOPAC>2.0.CO;2.

[23] M. Lukáčová-Medvid'ová, J. Rosemeier, P. Spichtinger, and B. Wiebe. IMEX finite volume methods for cloud simulation. In Finite volumes for complex applications VIII-hyperbolic, elliptic and parabolic problems, volume 200 of Springer Proc. Math. Stat., pages 179-187. Springer, Cham, 2017.

[24] Peter J. Marinescu, Susan C. van den Heever, Stephen M. Saleeby, Sonia M. Kreidenweis, and Paul J. DeMott. The microphysical roles of lower-tropospheric versus midtropospheric aerosol particles in mature-stage mcs precipitation. Journal of the Atmospheric Sciences, 74(11):3657-3678, NOV 2017. 10.1175/JAS-D-16-0361.1. 
[25] A.W. Marshall, I. Olkin, and B. C. Arnold. Inequalities: Theory of Majorization and Its Applications. Springer Series in Statistics. Springer, 2011. ISBN 978-0-387-40087-7. 10.1007/978-0-387-68276-1.

[26] J. S. Marshall and W. McK. Palmer. The distributions of raindrops with size. Journal of Meteorology, 5:165-166, 1948.

[27] H. Morrison and Wojciech W. Grabowski. Comparison of bulk and bin warm-rain microphysics models using a kinematic framework. Journal of the Atmospheric Sciences, 64(8):2839-2861, 2007. 10.1175/JAS3980. URL http://dx.doi.org/10.1175/ JAS3980.

[28] H. Morrison, J. A. Curry, and V. I. Khvorostyanov. A new double-moment microphysics parameterization for application in cloud and climate models. part i: Description. Journal of the Atmospheric Sciences, 62(6):1665-1677, 2005. 10.1175/JAS3446.1. URL https://doi.org/10.1175/JAS3446.1.

[29] Hugh Morrison and Wojciech W. Grabowski. Modeling supersaturation and subgrid-scale mixing with two-moment bulk warm microphysics. Journal of the Atmospheric Sciences, 65(3):792-812, 2008. 10.1175/2007JAS2374.1.

[30] Hugh Morrison and Jason A. Milbrandt. Parameterization of cloud microphysics based on the prediction of bulk ice particle properties. part i: Scheme description and idealized tests. Journal of the Atmospheric Sciences, 72(1):287-311, 2015. 10.1175/JAS-D-14-0065.1.

[31] DM Murphy and T Koop. Review of the vapour pressures of ice and supercooled water for atmospheric applications. Quarterly Journal of the Royal Meteorological Society, 131(608, B):1539-1565, 2005. 10.1256/qj.04.94.

[32] Ann Kristin Naumann and Axel Seifert. A lagrangian drop model to study warm rain microphysical processes in shallow cumulus. Journal of Advances in Modeling Earth Systems, 7(3):1136-1154, 2015. 10.1002/2015MS000456.

[33] M. D. Petters and S. M. Kreidenweis. A single parameter representation of hygroscopic growth and cloud condensation nucleus activity. Atmospheric Chemistry and Physics, 7(8):1961-1971, 2007. 10.5194/acp-7-1961-2007.

[34] H.R. Pruppacher and J.D Klett. Microphysics of Clouds and Precipitation. Springer, 2010. 10-1007/978-0-306-48100-0.

[35] H.R. Pruppacher and R. Rasmussen. Wind-tunnel investigation of the rate of evaporation of large water drops falling at terminal velocity in air. Journal of the Atmospheric Sciences, 36(7):1255-1260, $1979 . \quad 10.1175 / 1520$ 0469(1979)036<1255:AWTIOT>2.0.C0;2.

[36] A. Seifert, U. Blahak, and R. Buhr. On the analytic approximation of bulk collision rates of non-spherical hydrometeors. Geoscientific Model Development, 7(2):463-478, 2014. 10.5194/gmd-7-463-2014.

[37] Axel Seifert and Klaus Dieter Beheng. A two-moment cloud microphysics parameterization for mixed-phase clouds. Part I: Model description. . Meteorology and Atmospheric Physics, 92:45-66, 2006.

[38] U. Wacker. Structual stability in cloud physics using parameterized microphysics. Beiträge zur Physik der Atmosphäre, 65: 231-242, 1992.

[39] U Wacker and A Seifert. Evolution of rain water profiles resulting from pure sedimentation: Spectral vs. parameterized description. Atmospheric Research, 58(1):19-39, 2001. 10.1016/S0169-8095(01)00081-3.

[40] W Walter. Ordinary differential equations. Graduate texts in mathematics. Springer, 1998. ISBN 978-1-4612-6834-5. 10.1007/978-1-4612-0601-9.

[41] J. Warner. The microstructure of cumulus cloud. part i. general features of the droplet spectrum. Journal of the Atmospheric Sciences, 26(5):1049-1059, 1969. 10.1175/1520-0469(1969)026<1049:TMOCCP>2.0.CO;2. URL http://dx.doi.org/10.1175/ 1520-0469(1969)026<1049:TMOCCP>2.0.CO;2.

[42] Manfred Wendisch, Ulrich Pöschl, Meinrat O. Andreae, Luiz A. T. Machado, Rachel Albrecht, Hans Schlager, Daniel Rosenfeld, Scot T. Martin, Ahmed Abdelmonem, Armin Afchine, Alessandro C. Araùjo, Paulo Artaxo, Heinfried Aufmhoff, Henrique M. J. Barbosa, Stephan Borrmann, Ramon Braga, Bernhard Buchholz, Micael Amore Cecchini, Anja Costa, Joachim Curtius, Maximilian Dollner, Marcel Dorf, Volker Dreiling, Volker Ebert, André Ehrlich, Florian Ewald, Gilberto Fisch, Andreas Fix, Fabian Frank, Daniel Fütterer, Christopher Heckl, Fabian Heidelberg, Tilman Hüneke, Evelyn Jäkel, Emma Järvinen, Tina Jurkat, Sandra Kanter, Udo Kästner, Mareike Kenntner, Jürgen Kesselmeier, Thomas Klimach, Matthias Knecht, Rebecca Kohl, Tobias Kölling, Martina Krämer, Mira Krüger, Trismono Candra Krisna, Jost V. Lavric, Karla Longo, Christoph Mahnke, Antonio 0. Manzi, Bernhard Mayer, Stephan Mertes, Andreas Minikin, Sergej Molleker, Steffen Münch, Björn Nillius, Klaus Pfeilsticker, Christopher Pöhlker, Anke Roiger, Diana Rose, Dagmar Rosenow, Daniel Sauer, Martin Schnaiter, Johannes Schneider, Christiane Schulz, Rodrigo A. F. de Souza, Antonio Spanu, Paul Stock, Daniel Vila, Christiane Voigt, Adrian Walser, David Walter, Ralf Weigel, Bernadett Weinzierl, Frank Werner, Marcia A. Yamasoe, Helmut Ziereis, Tobias Zinner, and Martin Zöger. Acridicon-chuva campaign: Studying tropical deep convective clouds and precipitation over amazonia using the new german research aircraft halo. Bulletin of the American Meteorological Society, 97(10):1885-1908, 2016. 10.1175/BAMS-D-14-00255.1. URL https://doi.org/10.1175/BAMS-D-14-00255.1. 